\title{
Study of the properties and spectral energy distributions of the Herbig AeBe stars HD 34282 and HD 141569^
}

\author{
B. Merín ${ }^{1}$, B. Montesinos ${ }^{1,2}$, C. Eiroa ${ }^{3}$, E. Solano ${ }^{1}$, A. Mora ${ }^{3}$, P. D’ Alessio ${ }^{4}$, N. Calvet ${ }^{5}$, \\ R. D. Oudmaijer ${ }^{6}$, D. de Winter ${ }^{7}$, J. K. Davies ${ }^{8}$, A. W. Harris ${ }^{9}$, A. Cameron ${ }^{10}$, H. J. Deeg ${ }^{11}$, R. Ferlet ${ }^{12}$, \\ F. Garzón ${ }^{11}$, C. A. Grady ${ }^{13}$, K. Horne ${ }^{10}$, L. F. Miranda ${ }^{2}$, J. Palacios ${ }^{3}$, A. Penny ${ }^{14}$, A. Quirrenbach ${ }^{15}$, \\ H. Rauer ${ }^{9}$, J. Schneider ${ }^{16}$, and P. R. Wesselius ${ }^{17}$ \\ ${ }^{1}$ Laboratorio de Astrofísica Espacial y Física Fundamental (LAEFF), Apartado 50727, 28080 Madrid, Spain \\ 2 Instituto de Astrofísica de Andalucía-CSIC, Apartado 3004, 18080 Granada, Spain \\ 3 Departamento de Física Teórica, Módulo C-XI, Facultad de Ciencias, Universidad Autónoma de Madrid, \\ 28049 Cantoblanco, Madrid, Spain \\ ${ }^{4}$ Centro de Radioastronomía y Astrofísica (UNAM), Apartado Postal 3-72 (Xangari), 58089 Morelia, Mexico \\ 5 Harvard-Smithsonian Center for Astrophysics, 60 Garden Street, Cambridge, MA 02138, USA \\ ${ }^{6}$ Department of Physics and Astronomy, University of Leeds, Leeds LS2 9JT, UK \\ 7 TNO/TPD-Space Instrumentation, Stieltjesweg 1, PO Box 155, 2600 AD Delft, The Netherlands \\ 8 Astronomy Technology Centre, Royal Observatory, Blackford Hill, Edinburgh EH9 3HJ, UK \\ 9 DLR Department of Planetary Exploration, Rutherfordstrasse 2, 12489 Berlin, Germany \\ 10 Department of Physics and Astronomy, University of St. Andrews, North Haugh, St. Andrews KY16 9SS, Scotland, UK \\ 11 Instituto de Astrofísica de Canarias, c/Via Láctea s/n, 38200 La Laguna, Tenerife, Spain \\ 12 CNRS, Institute d'Astrophysique de Paris, 98bis Bd. Arago, 75014 Paris, France \\ 13 NOAO/STIS, Goddard Space Flight Center, Code 681, NASA/GSFC, Greenbelt, MD 20771, USA \\ 14 Rutherford Appleton Laboratory, Chilton, Didcot, Oxfordshire OX11 0QX, UK \\ 15 Sterrewacht Leiden, PO Box 9513, 2300 RA Leiden, The Netherlands \\ 16 Observatoire de Paris, Place Jules Janssen, 92195 Meudon, France \\ 17 SRON, Universiteitscomplex “Zernike”, Landleven 12, PO Box 800, 9700 AV Groningen, The Netherlands
}

Received 22 October 2003 / Accepted 10 February 2004

\begin{abstract}
We present a study of the stellar parameters, distances and spectral energy distributions (SEDs) of HD 34282 and HD 141569, two pre-main sequence Herbig AeBe stars. Both objects have been reported to show "anomalous positions" in the HR diagram in the sense that they appear below the main sequence. A significant result of this work is that both stars are metal-deficient. The Hipparcos distance of HD 34282 is very uncertain and the current study places the star at the expected evolutionary position in the HR diagram, i.e. as a PMS star. The distance for HD 141569 found in this work matches the Hipparcos distance, and the problem of its anomalous position is solved as a result of the low metallicity of the object: using the right metallicity tracks, the star is in the PMS region. The SEDs are constructed using data covering ultraviolet to millimetre wavelengths. Physical, non-parametric models, have been applied in order to extract some properties of the disks surrounding the stars. The disk around HD 34282 is accreting actively, it is massive and presents large grains in the mid-plane and small grains in the surface. HD 141569 has a very low mass disk, which is in an intermediate stage towards a debris-type disk.
\end{abstract}

Key words. stars: pre-main sequence - stars: fundamental parameters - stars: planetary systems: protoplanetary disks

\section{Introduction}

The study of protoplanetary disks is currently undergoing an exciting stage partly propelled by the discovery of extrasolar planetary systems following the detection of 51 Peg B by

Send offprint requests to: B. Merín, e-mail: bruno@laeff.esa.es

* Based on observations made with the CST, NOT, INT and WHT telescopes of the Canary Islands observatories under the auspices of its International Time Programme. Also based on observations made with the $2.2 \mathrm{~m}$ telescope at Calar Alto Observatory (Almería).
Mayor \& Queloz (1995). The possible discovery of telluric planets in the near future, in addition to the Jovian-like planets already detected, will pose interesting questions on the formation of extrasolar planetary systems. Knowledge of the properties of protoplanetary disks and how they evolve to debris disks around main-sequence (MS) stars is one of the tools required for modelling such a process.

Observed spectral energy distributions (SEDs) of pre-main sequence (PMS) stars are widely used to study the properties of protoplanetary disks and to classify $\mathrm{T}$ Tauri and 
Herbig AeBe stars (HAeBe hereafter) into an evolutionary scheme (Adams et al. 1987; Hillenbrand et al. 1992). Although the interpretation of a given SED is, to some extent, model dependent, the theoretical modelling of SEDs constitutes an invaluable tool for understanding the structure and properties of protoplanetary disks, e.g. Chiang \& Goldreich (1997, 1999), D'Alessio et al. (1998, 1999, 2001), Dullemond et al. (2001). The EXPORT consortium (Eiroa et al. 2000) observed a large sample of PMS and Vega-type stars during the 1998 International Time Programme of the Canary Islands' Observatories. One of the driving goals of this effort was the study of the evolution and properties of protoplanetary disks by analysing the SEDs of the young stars, taking advantage of the fact that the EXPORT optical and near-IR photometry were obtained simultaneously. This observational approach is appropriate since $\mathrm{T}$ Tauri and $\mathrm{HAeBe}$ stars vary markedly in these spectral regimes and a significant part of the total luminosity of the object is radiated by the PMS stellar photosphere at these wavelengths. Among the stars in the EXPORT sample with measured Hipparcos parallaxes, the HAeBe stars HD 34282 and HD 141569 are the only ones whose positions fall below the zero-age main sequence in a $\log L-\log T_{\text {eff }} H R$ diagram or equivalent (e.g. van den Ancker et al. 1998; Weinberger et al. 2000). This result is difficult to reconcile with some observational results and with theoretical PMS evolutionary models (e.g. Yi et al. 2001).

In this paper we present an analysis of the stellar properties of HD 34282 and HD 141569 and their circumstellar disks, based on EXPORT data complemented with new spectroscopic observations and data from the literature. The structure of the paper is as follows. In Sect. 2 we briefly review what is currently known about the stars. Sections 3 and 4 present the observations used in this work and their results. In Sect. 5 we do a comprehensive study of the stellar parameters and distances to the stars. In Sect. 6 the disk models used to reproduce the observed SEDs are presented. In Sect. 7 we summarize the results of the work.

\section{The stars}

$H D$ 34282: it is a HAeBe star with spectral type estimates in the range $\mathrm{A} 0 \mathrm{~V}-\mathrm{A} 3 \mathrm{~V}$ and a rotational velocity of $v \sin i=$ $129 \pm 8 \mathrm{~km} \mathrm{~s}^{-1}$ (Mora et al. 2001). It is variable with published $m_{V}$ values between 9.8 and $10.11 \mathrm{mag}$ (Sylvester et al. 1996; de Winter et al. 2001). Malfait et al. (1998) report a visual variability amplitude of $\sim 2.5 \mathrm{mag}$, while an Algol-type minimum of $\Delta m \sim 0.8 \mathrm{mag}$ is observed in the Hipparcos light curve, indicating that HD 34282 is most likely an UXOR-type object. Its SED shows a strong IR excess which is already noticeable at near-IR wavelengths (Sylvester et al. 1996; Malfait et al. 1998). The Hipparcos parallax is $\pi=6.10 \pm 1.63$ mas, equivalent to a distance of $164_{-30}^{+60} \mathrm{pc}$. Based on this distance, van den Ancker et al. (1998) estimate a luminosity of $4.8 L_{\odot}$, a value which locates HD 34282 in an anomalous position in the HR diagram, well below the expected luminosity of a star of its spectral type. Note that the error in the parallax, namely $\sigma=1.63 \mathrm{mas}$, is slighly larger than usual for Hipparcos objects, most probably due to the faintness of the star, affecting the estimate of the distance. A large Keplerian disk around HD 34282 has been inferred from interferometric ${ }^{12} \mathrm{CO} J=2 \rightarrow 1$ line observations (Piétu et al. 2003). Those authors estimate a distance $d=400_{-100}^{+170} \mathrm{pc}$ based on dynamical considerations, which yields $L_{*} / L_{\odot}=29_{-13}^{+30}$ and $M_{*} / M_{\odot}=2.1_{-0.2}^{+0.4}$ for the star, much more consistent with the expected stellar properties of HD 34282.

$H D$ 141569: this star is considered to be a transitional object between the PMS HAeBe stars and the more evolved Vegatype systems. Optical and near-IR images reveal a circumstellar disk with a complex annular structure extending up to a radial distance of about $600 \mathrm{AU}$ (Weinberger et al. 1999; Augereau et al. 1999; Mouillet et al. 2001; Clampin et al. 2003). Mid-IR images trace the inner portion of the disk, up to about $100 \mathrm{AU}$ (Fisher et al. 2000; Marsh et al. 2002). The disk in HD 141569 shares many of the properties of dusty debris disks associated with young MS stars, such as $\beta$ Pic or HR 4796. On the other hand, the gas content of the disk is significantly higher than that of MS debris disks, as evidenced by the $\mathrm{CO}$ measurements at millimetre and near-IR wavelengths (Zuckerman et al. 1995; Brittain \& Rettig 2002; Boccaletti et al. 2003).

Concerning the star itself, HD 141569 has spectral type estimates between $\mathrm{B} 9$ and $\mathrm{A} 0 \mathrm{~V}$ and a very high rotational velocity, $v \sin i=258 \pm 17 \mathrm{~km} \mathrm{~s}^{-1}$ (Andrillat et al. 1990; Mora et al. 2001, and references therein). The star has no photometric variability (Alvarez \& Schuster 1981). Its spectrum exhibits double-peaked $\mathrm{H} \alpha$ emission superimposed on a broad and strong absorption line (Andrillat et al. 1990; Dunkin et al. 1997) as well as double-peaked emission of O I at 7772 and $8446 \AA$ (Andrillat et al. 1990); an outflow of material is suggested by the Na I D lines (Dunkin et al. 1997). Van den Ancker et al. (1998) estimate an age $>10^{7}$ years, while Weinberger et al. (2000) give an age of $5 \mathrm{Myr}$, on the basis of the stellar properties and age estimates of two nearby stars, which are most likely its companions and have $\mathrm{T}$ Tauri characteristics. Considering the Hipparcos distance, 99 pc, and the observed optical brightness and colours (e.g. de Winter et al. 2001), we deduce an absolute magnitude of $M_{V}=1.8 \mathrm{mag}$, assuming an $\mathrm{A} 0 \mathrm{~V}$ spectral type and a typical interstellar extinction law (Rieke \& Lebofsky 1985). This makes the star underluminous, a property seemingly shared by the debris disk systems $\beta$ Pic, HR 4796 and some other A-type young MS stars (Jura et al. 1998; Lowrance et al. 2000). We note, however, that HD 141569 has the largest IR excess among all these objects, it is the only object with emission lines in its optical spectrum, there is evidence of outflowing gas, its disk has a remarkable gas content, and it likely has the smaller evolutionary age. In addition, from a PMS evolution theory point of view, a $5 \mathrm{Myr}$ old A0 star $\left(T_{\text {eff }}=10000 \mathrm{~K}\right.$, see below) with solar metallicity would have an absolute magnitude $M_{V}=1.0-1.1 \mathrm{mag}$ (e.g. Yi et al. 2001), which is significantly brighter than the magnitude deduced from the Hipparcos parallax.

\section{Observations}

High resolution echelle (wavelength range 3800-5900 A) and intermediate resolution (wavelength range 5800-6700 $)$ ) spectroscopy, optical photo-polarimetry and near-infrared 
Table 1. Telescopes, instruments, setups and observing modes.

\begin{tabular}{lllll}
\hline \hline Telescope & Instrument & Range/Bands & $\lambda / \Delta \lambda$ & Observing mode \\
\hline WHT $(4.2 \mathrm{~m})$ & UES & $3800-5900 \AA$ & $\sim 49000$ & Echelle spectroscopy \\
INT $(2.5 \mathrm{~m})$ & IDS & $5800-6800 \AA$ & $\sim 5000$ & Mid resolution spectroscopy \\
NOT $(2.5 \mathrm{~m})$ & Turpol (photopol.) & $U B V R I$ & & Photopolarimetry \\
CST $(1.5 \mathrm{~m})$ & Photometer+near-IR camera & $J H K$ & & Photometry \\
CAHA $2.2 \mathrm{~m}$ & CAFOS (spectros.) & $3700-6440 \AA$ & $\sim 2400$ & Mid resolution spectroscopy \\
\hline
\end{tabular}

Observing dates for the spectroscopic observations ${ }^{\dagger}$.

\begin{tabular}{lll}
\hline \hline & HD 34282 & HD 141569 \\
\hline WHT/UES & 28, 29 January 99 & 16, 17 May 98 (2+2) \\
& & 28, 29, 30 July 98 \\
& & 28, 29, 30, 31 January 99 \\
\hline INT/IDS & $24,25,26,27,28$ October 98 & $14,15,16,17$ May 98 \\
& $29,30,31$ January 99 & 29, 30, 31 July 98 \\
& & 29, 30, 31 January 99 \\
\hline CAHA 2.2/CAFOS & 3 February 03 & 6 March 03 \\
\hline
\end{tabular}

$\dagger$ The observing logs for the photometric observations are given by Eiroa et al. (2001) and Oudmaijer et al. (2001).

photometry of HD 34282 and HD 141569 were obtained during the four EXPORT observational campaigns in May, July and October 1998 and January 1999 carried out at the Canary Islands observatories (Eiroa et al. 2000). Details of the observations, instrumental setups and reduction procedures for each particular observing mode are given in Eiroa et al. (2001), Mora et al. (2001) and Oudmaijer et al. (2001), so we do not repeat them here, though Table 1 provides an overview of the telescopes, instruments, setups and observing modes used during the EXPORT campaigns, plus the additional observations carried out in 2003 (see below). The dates for the spectroscopic observations are also specified.

The spectra of HD 34282 and HD 141569 are presented and analysed here for the first time, while the photometric data, though already published as such in the previous references, are collected here together and also discussed for the first time. We note that the data are simultaneous or almost simultaneous, allowing a coherent study to be made of the photometric and spectroscopic variations, and the construction of the corresponding SEDs in the interval UBVRIJHK with contemporaneous data.

In addition to the EXPORT observations, intermediate resolution spectra in the range 3700-6200 $\AA$, with very high signal-to-noise ratio, have been taken for both stars with CAFOS (Calar Alto Faint Object Spectrograph) on the $2.2 \mathrm{~m}$ telescope at Calar Alto observatory (Almería, Spain). The motivation was to obtain high quality Balmer line profiles, in particular $\mathrm{H} \beta, \mathrm{H} \gamma$ and $\mathrm{H} \delta$, in order to estimate the stellar gravities (see Sect. 5). The echelle spectra were inadequate for analysis of the Balmer line profiles: these are so wide that their wings extend up to three echelle spectral orders - the correction of the response function of the spectrograph and the splicing of adjacent orders are not accurate enough to consider the reconstructed profiles as reliable. CAFOS was equipped with a CCD SITe detector of $2048 \times 2048$ pixels (pixel size $24 \mu \mathrm{m}$ ) and the grism Blue-100, centered at $4238 \AA$, giving a reciprocal linear dispersion of $88 \AA / \mathrm{mm}(2 \AA /$ pixel). Three spectra, with exposure times 120, 1200 and $1200 \mathrm{~s}$, were taken for HD 34282 on 5 February 2003, and two with exposure times 10 and $100 \mathrm{~s}$ were obtained for HD 141569 on 6 March 2003. The usual bias, dark and dome flat-field frames were taken in each case. Standard procedures were used to process the data. The nominal wavelengths of the Balmer lines were used to self-calibrate the spectra in wavelength.

\section{Results}

\subsection{Spectroscopy}

The high and intermediate resolution spectra of both stars show non-variable early-type photospheric spectra, interstellar (IS) narrow absorption lines and diffuse IS bands. In both cases, however, the most prominent features come from the circumstellar (CS) environment. Figure 1 shows the average INT spectra of HD 34282 (January 99) and HD 141569 (May 98) where some lines are identified. In the following, we describe the CS spectrum of both objects.

$H D$ 34282: the UES echelle spectra show a CS contribution in the $\mathrm{Ca}$ II $\mathrm{K}$ line and in the Balmer lines $\mathrm{H} \beta$ and $\mathrm{H} \gamma$. The line $\mathrm{He}$ I at $5876 \AA$ is seen in absorption; it is unlikely to be of photospheric origin since HD 34282 has an $\sim A 3$ spectral type. Its strength and profile might show small variations (after a comparison of all the INT spectra), but this should be confirmed with higher resolution spectra. He I $5876 \AA$ has been observed with large variations in HAeBe and T Tauri stars (e.g. Böhm \& Catala 1995; Johns \& Basri 1995) and it has been explained in terms of magnetospheric accretion in the low-mass PMS stars (e.g. Oliveira et al. 2000), but in the case of HAeBe stars its origin is controversial (e.g. Bouret \& Catala 2000).

$\mathrm{H} \alpha$ always presents a variable double-peaked emission with a central absorption. Figure 2 shows the observed $\mathrm{H} \alpha$ profiles 


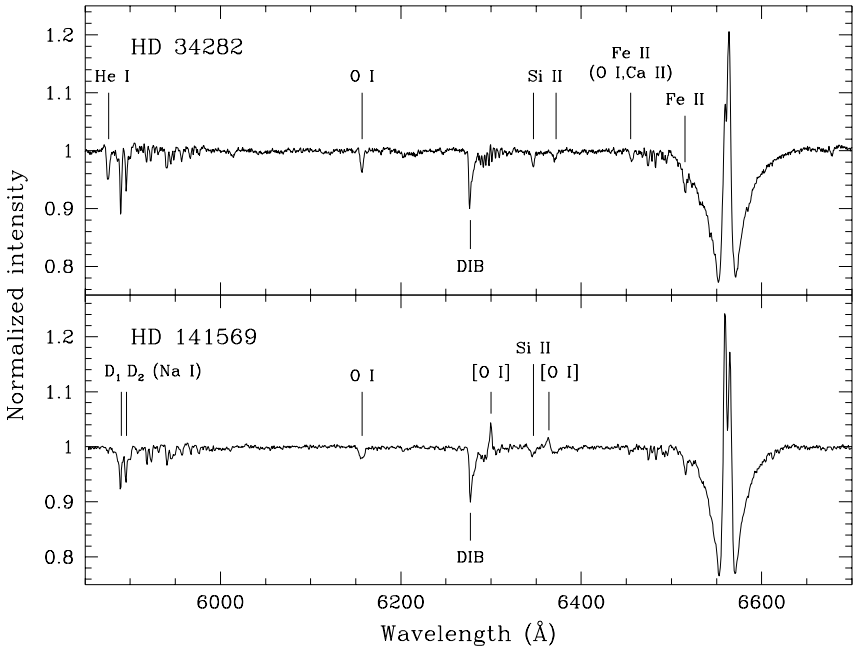

Fig. 1. The normalized average IDS/INT spectra of HD 34282 and HD 141569. Some prominent lines are identified. DIB stands for "diffuse interstellar band". The spectrum of HD 34282 is the average of the January 1999 run while the spectrum of HD 141569 is the average of the May 1998 run.

after the subtraction of a spectrum of a standard of the same spectral type broadened to the rotational velocity of HD 34282. The depth of the central absorption varies and its radial velocity, measured with respect to the stellar photosphere, shifts from an average value of $27.5 \pm 5.5 \mathrm{~km} \mathrm{~s}^{-1}$ in October 98 to $-63.3 \pm 14.6 \mathrm{~km} \mathrm{~s}^{-1}$ in January 99. The radial velocities of the violet and red emission peaks and their separation also change from October 98 to January 99 (the maximum peak separation is $319 \mathrm{~km} \mathrm{~s}^{-1}$ on 28 Oct. 98 and the minimum is $117 \mathrm{~km} \mathrm{~s}^{-1}$ on 30 Jan. 99). The relative intensity of the emission peaks changes from day to day and a flip is observed from October 98, when the violet peak is stronger, to January 99, when the read peak is the stronger one. Furthermore, the blue wing shows a shoulder on 28 October 98 which could be due to a third velocity component. Finally, the total equivalent width varies from -3.0 to $-5.7 \AA$. Using the $R$ band flux from simultaneous optical photometry on 27 October 98 and 30 January 99 we estimate a $\mathrm{H} \alpha$ line emission flux of $7.4 \times 10^{-13}$ and $1.6 \times 10^{-12} \mathrm{erg} \mathrm{cm}^{-2} \mathrm{~s}^{-1}$, respectively. The variability in the emission flux and shape of $\mathrm{H} \alpha$ proves the changing conditions in the CS region where this line originates. Grinin \& Rostopchina (1996) studied a sample of HAeBe stars and found that double-peaked $\mathrm{H} \alpha$ emission likely arises in irregular gaseous circumstellar disks rotating close to the stars and seen nearly edge on. We notice that Piétu et al. (2003) find an inclination of the $\mathrm{CO}$ Keplerian disk of $i=56^{\circ}$ with respect to the plane of the sky. The change of the strength ratio between the violet and red peaks could reflect the relative contribution of the approaching and receding parts of the disk in the different spectra. More sophisticated accretion disk models have recently been developed by Tambovtseva \& Grinin (2000) to explain the hydrogen lines of HAeBe stars in a context similar to the widely accepted magnetospheric accretion models for the low-mass $\mathrm{T}$ Tauri stars.
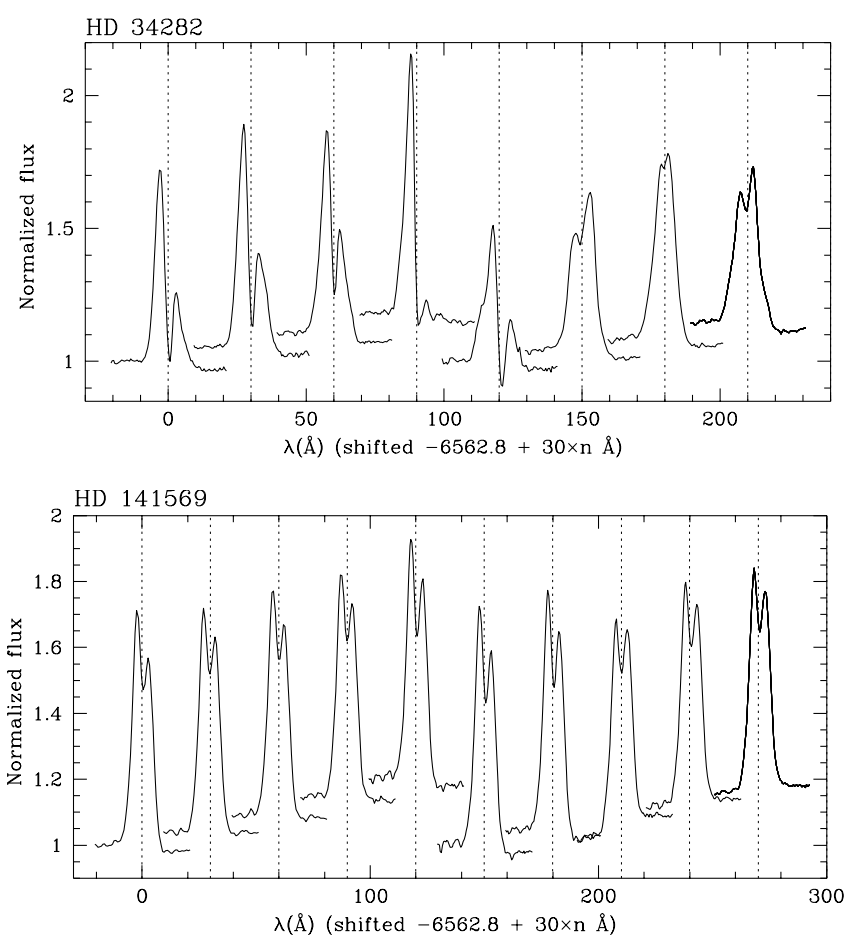

Fig. 2. H $\alpha$ profiles for HD 34282 and HD 141569. Spectra of standard stars with spectral types A3 V and A0 V, respectively, have been subtracted from the original spectra, therefore only the pure emission is shown in these panels. The lines have been shifted vertically by an arbitrary amount for clarity, so their wings should be put at the same level in order to compare the profiles. Each profile has been shifted horizontally $30 \AA$ with respect to the previous one as indicated in the abscissae. The vertical dotted lines mark the rest wavelength for $\mathrm{H} \alpha$. The spectra have been ordered chronologically from left to right (see the dates of the observations in Table 1).

HD 141569: double-peaked $\mathrm{H} \alpha$ emission is observed in the spectrum of this star. Such double-peaked emission, though much less pronounced, is also observed superimposed to the $\mathrm{H} \beta$ photospheric line in all UES spectra. The heliocentric radial velocity of the star obtained from the UES spectra is $13.1 \mathrm{~km} \mathrm{~s}^{-1}$, which is in good agreement with previous estimates (Dunkin et al. 1997; Brittain et al. 2003), given the velocity resolution of the UES spectra, $6 \mathrm{~km} \mathrm{~s}^{-1}$. The violet and red $\mathrm{H} \alpha$ emission peaks have $-113.2 \pm 3.9 \mathrm{~km} \mathrm{~s}^{-1}$ and $128.3 \pm 5.0 \mathrm{~km} \mathrm{~s}^{-1}$, respectively, while the central absorption lies at a velocity of $11.9 \pm 4.2$ (average values and rms error of the 10 INT spectra, velocities measured with respect to the stellar photosphere). Dunkin et al. (1997) find agreement between the stellar and the $\mathrm{H} \alpha$ central absorption velocities; the discrepancy in our values must be treated with caution given the spectral resolution of the individual INT spectra, $\sim 50 \mathrm{~km} \mathrm{~s}^{-1}$. The observed velocity separation between both emission peaks is in good agreement with the results of Dunkin et al. (1997). The emission profiles are quite similar in all spectra, although they vary noticeably. The violet peak is always stronger than the red one, but their relative intensity changes (Fig. 2); there is also a variation in the $\mathrm{H} \alpha$ blue wing (Fig. 3). In addition, the equivalent width varies, ranging from $-6.14 \pm 0.03 \AA$ in July 1998 , when the violet peak was larger, to $-5.63 \pm 0.25 \AA$ 
Table 2. Compilation of optical (UBVRI) and near-IR (JHK) photometry for HD 34282 and HD 141569.

\begin{tabular}{lccccccccc}
\hline \hline Object & $U$ & $B$ & $V$ & $R$ & $I$ & $J$ & $H$ & $K$ & Refs. \\
\hline HD 34282 & 10.47 & 10.23 & 10.03 & 9.91 & 9.76 & 9.11 & 8.40 & 7.62 & 1, October 98 \\
& 10.19 & 10.03 & 9.87 & 9.77 & 9.63 & 9.15 & 8.53 & 7.84 & 1 , January 99 \\
& & & 10.11 & & & 9.17 & 8.32 & 7.42 & 2 \\
& 10.16 & 10.05 & 9.88 & 9.79 & 9.68 & 9.02 & 8.20 & 7.44 & 3 \\
& 10.15 & 10.05 & 9.89 & 9.81 & 9.71 & & & & 4 \\
& 10.11 & 9.97 & 9.81 & 9.73 & 9.62 & & & & 4 \\
& 10.51 & 10.07 & 9.94 & 9.95 & 9.79 & & & & 4 \\
& & & & & & 8.99 & 8.54 & 8.08 & 6 \\
\hline HD 141569 & 7.23 & 7.20 & 7.11 & 7.01 & 7.03 & 6.70 & 6.59 & 6.53 & 1 \\
& & & 7.09 & & & 6.88 & 6.86 & 6.82 & 2 \\
& & & 7.13 & & & 6.87 & 6.84 & 6.80 & 3 \\
& 7.24 & 7.21 & 7.10 & 7.03 & 6.96 & & & & 4 \\
\hline
\end{tabular}

(1) EXPORT; (2) Malfait et al. (1998), (3) Sylvester et al. (1996), (4) de Winter et al. (2001), (5) Penprase (1992), (6) Coulson et al. (1998).

in January 1999, when the violet peak was smaller. The average $\mathrm{H} \alpha$ line flux is $2.1 \times 10^{-11} \mathrm{erg} \mathrm{cm}^{-2} \mathrm{~s}^{-1}$.

All INT spectra show [O I] in emission, relatively strong at $6300 \AA$ and very weak at $6364 \AA$. The emission is constant and has an equivalent width $W([\mathrm{O}$ I] $6300 \AA)=0.13 \AA$; its flux is $4.6 \times 10^{-13} \mathrm{erg} \mathrm{cm}^{-2} \mathrm{~s}^{-1}$. The peak of the emission occurs at the same radial velocity as the $\mathrm{H} \alpha$ absorption peak. [O I] emission is usually interpreted as an indicator of low density winds in HAeBe stars (Böhm \& Catala 1994; Corcoran \& Ray 1997).

\subsection{Photometry and the spectral energy distribution}

EXPORT optical UBVRI and near-IR JHK magnitudes of HD 32482 and HD 141569 are given in Oudmaijer et al. (2001) and Eiroa et al. (2001). HD 34282 varies in both wavelength regimes from October 98 to January 99 , but no variability is detected on time scales of days. HD 141569 remains constant on all time scales within the photometric uncertainties (typically a few percents in any photometry band). Table 2 shows the mean magnitudes of HD 34282 as observed in October 98 and January 99, as well as the mean magnitudes of HD 141569. For the sake of comparison, photometric results taken from the literature are also shown. In general, typical intrinsic error bars in the EXPORT UBVRI magnitudes are of the order of $\pm 0.04 \mathrm{mag}$ and always less than $0.10 \mathrm{mag}$; for $J H K$ they are less than 0.05 mag (see Eiroa et al. 2001; Oudmaijer et al. 2001 for specific values).

HD 34282 was brighter in the optical in January 99 than in October 98 (the values of $V$ are 10.02, 10.03 and 9.87 for 24, 28 October 98 and 30 January 99, respectively). The January 99 magnitudes likely correspond to the maximum optical brightness level of this star, as comparison with other results (Table 2) and with the Hipparcos light curve suggests. The near-IR brightness behaves in the opposite way: the star was brighter in October 98. The optical variability amplitude decreases with wavelength, as expected from the usual

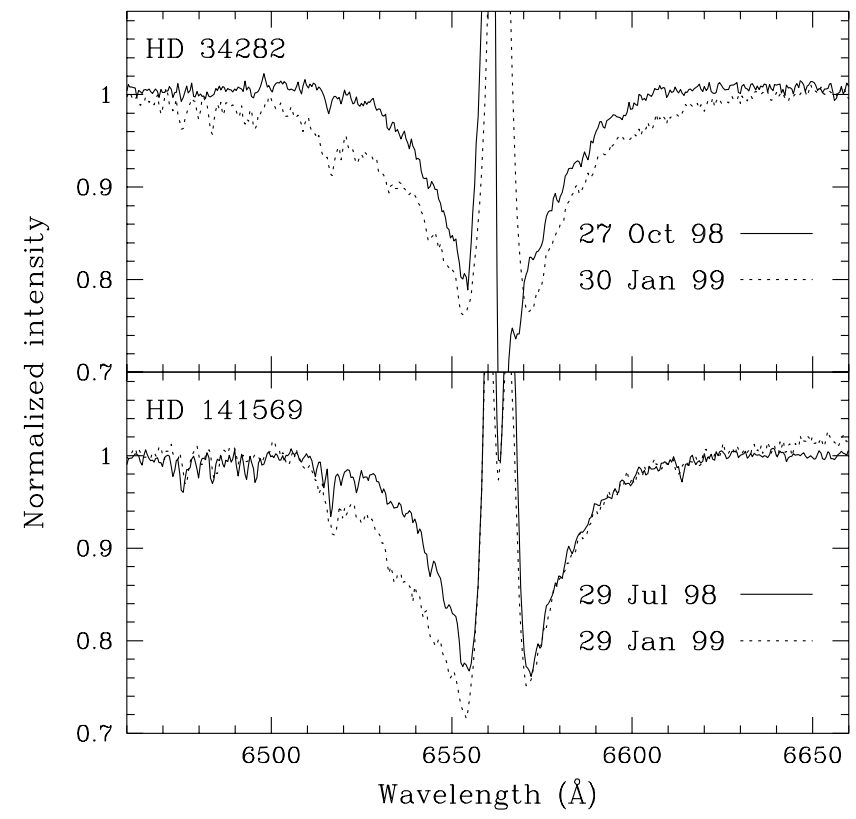

Fig. 3. Details of the $\mathrm{H} \alpha$ profiles for HD 34282 and HD 141569. The most extreme cases have been taken for each star to illustrate the complex behaviour of this line. The variations in the absorption profiles appear in both wings but are more pronounced in the blue one.

mechanisms invoked to explain the photometric variability in PMS stars (e.g. Herbst \& Shevchenko 1999), but in the near-IR the maximum variability amplitude occurs in $K$. The cause of this different behaviour might be due to the fact that the optical variability is due to changes affecting the stellar photosphere while the near-IR fluxes reflect the contribution of the star and the circumstellar disk. A simultaneous anticorrelation of the variability in both wavelength regimes has also been found in 


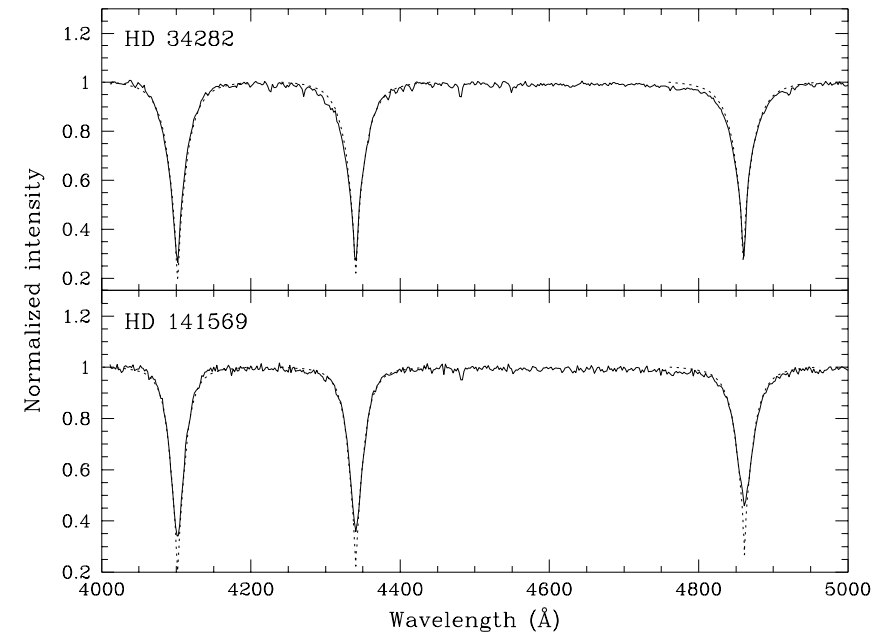

Fig. 4. The Balmer lines $\mathrm{H} \beta, \mathrm{H} \gamma$ and $\mathrm{H} \delta$ for $\mathrm{HD} 34282$ and $\mathrm{HD} 141569$ (solid lines) extracted from the spectra taken with CAFOS on the $2.2 \mathrm{~m}$ telescope at CAHA. The dotted lines are the Kurucz synthetic profiles corresponding to the $T_{\text {eff }}, \log g_{*}$ and metallicity listed in Table 4 for each star. The discrepancies in the higher parts of the wings arise from contamination with weak metalic lines and also from the difficulties in normalizing the original spectra.

other pre-main sequence stars, though on a much shorter time scale (Eiroa et al. 2002; Grinin et al. 2002).

Concerning HD 141569, our optical photometry and previous results are in excellent agreement. The apparent discrepancy between our near-IR magnitudes and those obtained by Sylvester et al. (1996) and Malfait et al. (1998) is likely due to the fact that the companion star HD 141569B was at least partly in our beam (Weinberger et al. 2000). Once our measurements are corrected for this, the agreement is satisfactory.

For both stars EXPORT photometry together with data from near ultraviolet to millimetre wavelengths have been used to delineate the spectral energy distributions, which are, to our knowledge, the most complete ones to date. Figures 9 and 10 show the observed spectral energy distributions of both stars. Table 3 gives dereddened fluxes for each star and corresponding references (they are also plotted in Figs. 9 and 10). $E(B-V)$ values of 0.05 for HD 34282 and 0.12 for HD 141569 (see Sect. 5.1 for the origin of these values) were used for the reddening corrections. The extintion curve of Rieke \& Lebofsky (1985), from $4400 \AA$ to longer wavelengths, and that of Steenman \& The (1991) for shorter wavelengths were used. We have used the UBVRI (one set of values) and the mean $J H K$ (three sets) photometry from January 99 for HD 34282 because they likely represent better the stellar photosphere, i.e. when the star was brighter in the optical. For HD 141569, we used the mean EXPORT UBVRI photometry and $J H K$ magnitudes from Malfait et al. (1998). UBVRIJHK magnitudes have been converted into fluxes using the absolute calibration given by Schmidt-Kaler (1982). IUE spectra in the range 1250-3000 ̊̊ of HD 141569 are taken from the INES database ${ }^{1}$ (Solano et al. 2001). No IUE observations are available for HD 34282. Walraven photometry

\footnotetext{
${ }^{1}$ http://ines.laeff.esa.es/
}

Table 3. Absolute dereddened fluxes for the SEDs.

\begin{tabular}{|c|c|c|c|}
\hline & & HD 34282 & HD 141569 \\
\hline Band & $\begin{array}{l}\log \lambda \\
(\mu \mathrm{m})\end{array}$ & $\begin{array}{c}\log F_{\lambda} \\
\left(\mathrm{W} \mathrm{m}^{-2} \mu \mathrm{m}^{-1}\right)\end{array}$ & $\begin{array}{c}\log F_{\lambda} \\
\left(\mathrm{W} \mathrm{m}^{-2} \mu \mathrm{m}^{-1}\right)\end{array}$ \\
\hline$I U E$ & -0.86 & - & $-9.90 \pm 0.02(4)$ \\
\hline IUE & -0.75 & - & $-9.79 \pm 0.02$ \\
\hline IUE & -0.66 & - & $-9.94 \pm 0.02(4)$ \\
\hline IUE & -0.59 & - & $-10.01 \pm 0.02(4)$ \\
\hline IUE & -0.53 & - & $-10.08 \pm 0.02(4)$ \\
\hline Wal W & -0.49 & $-11.45 \pm 0.02(5)$ & - \\
\hline Wal $U$ & -0.44 & $-11.44 \pm 0.02(5)$ & - \\
\hline Wal L & -0.42 & $-11.23 \pm 0.02(5)$ & - \\
\hline Wal B & -0.37 & $-11.11 \pm 0.02$ & - \\
\hline Wal V & -0.26 & $-11.32 \pm 0.02(5)$ & - \\
\hline$U$ & -0.44 & $-11.35 \pm 0.01$ & $-10.02 \pm 0.02(1)$ \\
\hline$B$ & -0.36 & $-11.12 \pm 0.01$ & $-9.88 \pm 0.02(1)$ \\
\hline$V$ & -0.26 & $-11.31 \pm 0.01$ & $-10.12 \pm 0.02(1)$ \\
\hline$R$ & -0.15 & $-11.62 \pm 0.01(1)$ & $-10.45 \pm 0.02(1)$ \\
\hline$I$ & -0.01 & $-11.90 \pm 0.01$ & $-10.82 \pm 0.04(1)$ \\
\hline$J$ & 0.10 & $-12.15 \pm 0.02$ & $-11.22 \pm 0.02(2)$ \\
\hline$H$ & 0.21 & $-12.32 \pm 0.02(1)$ & $-11.64 \pm 0.02(2)$ \\
\hline$K$ & 0.35 & $-12.54 \pm 0.02$ & $-12.12 \pm 0.02(2)$ \\
\hline ISO & 0.39 & - & $-12.27 \pm 0.02(6)$ \\
\hline ISO & 0.51 & - & $-12.66 \pm 0.02(6)$ \\
\hline$L$ & 0.58 & $-12.75 \pm 0.02$ & $-12.92 \pm 0.02(3)$ \\
\hline ISO & 0.63 & - & $-13.14 \pm 0.02(6)$ \\
\hline$M$ & 0.68 & $-13.12 \pm 0.02$ & $-13.25 \pm 0.02(3)$ \\
\hline ISO & 0.77 & - & $-13.46 \pm 0.02(6)$ \\
\hline ISO & 0.83 & - & $-13.54 \pm 0.02(6)$ \\
\hline ISO & 0.89 & - & $-13.39 \pm 0.02(6)$ \\
\hline ISO & 0.94 & - & $-13.61 \pm 0.02(6)$ \\
\hline ISO & 0.99 & - & $-13.78 \pm 0.02(6)$ \\
\hline ISO & 1.03 & - & $-13.82 \pm 0.02(6)$ \\
\hline ISO & 1.06 & - & $-13.84 \pm 0.02(6)$ \\
\hline IRAS & 1.08 & $-13.82 \pm 0.10(7)$ & $-13.92 \pm 0.05(7)$ \\
\hline IRAS & 1.40 & $-14.09 \pm 0.10(7)$ & $-14.03 \pm 0.07(7)$ \\
\hline IRAS & 1.78 & $-14.03 \pm 0.10(7)$ & $-14.32 \pm 0.09(7)$ \\
\hline IRAS & 2.00 & $-14.50 \pm 0.10(7)$ & $-15.00 \pm 0.10(7)$ \\
\hline $0.45 \mathrm{~mm}$ & 2.65 & $-16.72 \pm 0.23$ & - \\
\hline $0.8 \mathrm{~mm}$ & 2.90 & $-17.72 \pm 0.07$ & - \\
\hline $1.1 \mathrm{~mm}$ & 3.04 & $-18.34 \pm 0.09$ & $<-19.05(3)$ \\
\hline $1.3 \mathrm{~mm}$ & 3.12 & $-18.71 \pm 0.09(8)$ & - \\
\hline $1.35 \mathrm{~mm}$ & 3.13 & - & $-20.05 \pm 0.02(9)$ \\
\hline $2.6 \mathrm{~mm}$ & 3.41 & $-19.99 \pm 0.13(10)$ & - \\
\hline $3.4 \mathrm{~mm}$ & 3.53 & $-20.89 \pm 0.06(8)$ & - \\
\hline
\end{tabular}

(1) EXPORT; (2) Malfait et al. (1998), (3) Sylvester et al. (1996), (4) INES Database, (5) de Geus et al. (1990), (6) ISO PHOT-S, TDT No. 62701662, (7) IRAS PSC (colour corrected), (8) Piétu et al. (2003), (9) Sylvester et al. (2001), (10) Mannings \& Sargent (2000).

for HD 34282 was not contemporaneous with EXPORT observations and has only been used in Sect. 6.2.1 for a determination of the disk mass accretion rate. In the infrared, we have used colour-corrected IRAS fluxes and ISO scientifically validated "Off-Line Processing" products (OLP) taken from the Data Archive at the ISO Data Centre. Submillimetre and 
millimetre continuum fluxes have been taken from the references listed in Table 3; the inclusion of the continuum fluxes at these long wavelengths alleviates, to some extent, the uncertainties in the flux calibration of the infrared ISO pipelinereduced fluxes.

\section{Stellar parameters of the stars and their distances}

As we mentioned above, both stars have been reported to have anomalous positions in the HR diagram, falling below the main sequence. However, the observational data show that HD 34282 is a highly variable HAeBe star with an active CS environment. Concerning HD 141569, though its disk shares similarities with some debris disk systems, which are found to be located below the main sequence, the star does show observational properties which suggest an earlier evolutionary stage. In particular, our data show that the object retains a non-negligible CS activity, as evidenced by the [O I] emission and the variable $\mathrm{H} \alpha$ emission. Taking into account these considerations, both stars should be located above or on the main sequence, in better agreement with normal expectations from their photometric and spectroscopic properties, and stellar evolution theory. In this section we carry out a detailed study of the physical parameters of the stars leading us to re-consider their location in the HR diagram. The determination of the effective temperature, gravity and stellar metallicity will allow us, by using the appropriate set of evolutionary tracks and isochrones, to estimate the luminosity, mass and age of the stars, as well as their distances by means of the measured stellar fluxes.

\subsection{Stellar parameters: Effective temperature, gravity, metallicity and rotational velocity}

Kurucz (1993) model atmospheres are used to estimate the physical parameters describing the photospheres of HD 34282 and HD 141569. We use the following iterative procedure. First, an initial value of the stellar gravity is obtained from the comparison of synthetic profiles, computed with solar metallicity, with the observed $\mathrm{H} \beta, \mathrm{H} \gamma$ and $\mathrm{H} \delta$ profiles extracted from the CAFOS observations. For this purpose, a tentative value of $T_{\text {eff }}$, based on the spectral type of each star, is taken. The gravity is obtained by measuring the width of the observed Balmer profiles at one intensity level below the normalized continuum, namely $I=0.80$; this has been chosen to avoid potential artifacts in the wings near the continuum arising from the normalization of the original spectra. The widths of the Balmer lines are then compared with those measured on a grid of synthetic profiles computed for different gravities ${ }^{2}$. A linear interpolation is done taking the widths of each Balmer line as independent variables and the gravity as the unknown.

\footnotetext{
2 The convolution of the theoretical profiles with the instrumental response changes the results by a minute quantity. For HD 141569, which is the star with less intrinsic uncertainties in the determination of the gravity, the relative difference with the value in Table 4 is about $0.5 \%$.
}

Table 4. Stellar parameters determined.

\begin{tabular}{|c|c|c|}
\hline & \multicolumn{2}{|c|}{ HD 34282} \\
\hline Effective temperature & \multicolumn{2}{|c|}{$8625 \pm 200 \mathrm{~K}$} \\
\hline Spectral type & \multicolumn{2}{|c|}{ A3 V } \\
\hline$v \sin i$ & \multicolumn{2}{|c|}{$110 \pm 10 \mathrm{~km} \mathrm{~s}^{-1}$} \\
\hline Metallicity & \multicolumn{2}{|c|}{$[\mathrm{Fe} / \mathrm{H}]=-0.8 \pm 0.1$} \\
\hline $\log g_{*}$ & \multicolumn{2}{|c|}{$4.20 \pm 0.20$} \\
\hline$E(B-V)$ & \multicolumn{2}{|c|}{0.05} \\
\hline Stellar flux $\left(F_{*}\right)$ & \multicolumn{2}{|c|}{$(3.61 \pm 0.44) \times 10^{-12} \mathrm{~W} \mathrm{~m}^{-2}$} \\
\hline IR Excess $\left(F_{\text {IR }}\right)$ & \multicolumn{2}{|c|}{$(2.28 \pm 0.40) \times 10^{-12} \mathrm{~W} \mathrm{~m}^{-2}$} \\
\hline$\lambda_{\mathrm{IR}}$ & \multicolumn{2}{|c|}{$1.25 \mu \mathrm{m}$} \\
\hline & {$\left[ \pm \Delta \log g_{*}\right]$} & {$\left[ \pm \Delta T_{\mathrm{eff}}\right]$} \\
\hline$L / L_{\odot}$ & $13.64_{+12.02}^{-5.36}$ & $13.64_{-1.53}^{+1.78}$ \\
\hline Mass $\left(M_{\odot}\right)$ & $1.59_{+0.30}^{-0.07}$ & $1.59_{-0.05}^{+0.04}$ \\
\hline Age (Myr) & $6.41_{-2.58}^{+1.92}$ & $6.41_{+0.46}^{-0.41}$ \\
\hline \multirow[t]{2}{*}{ Distance (pc) } & $348_{+129}^{-77}$ & $348_{-20}^{+22}$ \\
\hline & \multicolumn{2}{|c|}{ HD 141569} \\
\hline Effective temperature & \multicolumn{2}{|c|}{$10000 \pm 200 \mathrm{~K}$} \\
\hline Spectral type & \multicolumn{2}{|c|}{ B9.5 V } \\
\hline$v \sin i$ & \multicolumn{2}{|c|}{$236 \pm 15 \mathrm{~km} \mathrm{~s}^{-1}$} \\
\hline Metallicity & \multicolumn{2}{|c|}{$[\mathrm{Fe} / \mathrm{H}]=-0.5 \pm 0.1$} \\
\hline $\log g_{*}$ & \multicolumn{2}{|c|}{$4.28 \pm 0.04$} \\
\hline$E(B-V)$ & \multicolumn{2}{|c|}{0.12} \\
\hline Stellar flux $\left(F_{*}\right)$ & \multicolumn{2}{|c|}{$(7.16 \pm 0.24) \times 10^{-11} \mathrm{~W} \mathrm{~m}^{-2}$} \\
\hline IR Excess $\left(F_{\mathrm{IR}}\right)$ & \multicolumn{2}{|c|}{$(1.27 \pm 0.27) \times 10^{-12} \mathrm{~W} \mathrm{~m}^{-2}$} \\
\hline \multirow[t]{2}{*}{$\lambda_{\mathrm{IR}}$} & \multicolumn{2}{|c|}{$3.25 \mu \mathrm{m}$} \\
\hline & {$\left[ \pm \Delta \log g_{*}\right]$} & {$\left[ \pm \Delta T_{\mathrm{eff}}\right]$} \\
\hline$L / L_{\odot}$ & $25.77_{+1.63}^{-2.20}$ & $25.77_{-2.54}^{+3.01}$ \\
\hline $\operatorname{Mass}\left(M_{\odot}\right)$ & $2.00_{-0.01}^{+0.01}$ & $2.00_{-0.05}^{+0.06}$ \\
\hline Age (Myr) & $4.71_{-0.15}^{+0.09}$ & $4.71_{+0.33}^{-0.03}$ \\
\hline Distance (pc) & $108_{+3}^{-5}$ & $108_{-6}^{+6}$ \\
\hline
\end{tabular}

The result for $\log g_{*}$ is the mean value of all the interpolations with an uncertainty corresponding to the standard deviation.

With this value of $\log g_{*}$ fixed, we re-estimate the efective temperatures of the stars and their metallicities. This is done by means of a systematic comparison of synthetic line profiles, obtained using Kurucz's models of different temperatures and metallicities, with the high resolution WHT/UES spectra. The regions of effective temperatures from 8500 to $9750 \mathrm{~K}$ for HD 34282, and from 9750 to $10500 \mathrm{~K}$ for HD 141569 were explored, which approximately encompass the values corresponding to the spectral types reported for both stars. For each temperature, different metallicities were used to synthesize spectral lines in several selected spectral intervals. The synthetic spectra were rotationally broadened with the corresponding $v \sin i$ values for each star (Mora et al. 2001). A microturbulence velocity of $2 \mathrm{~km} \mathrm{~s}^{-1}$ was used throughout.

For HD 34282 the lines chosen are: $4404.75,4957.60 \AA$ (Fe I $), 4508.29,4515.34,4583.84,4629.34 \AA$ (Fe II) and 


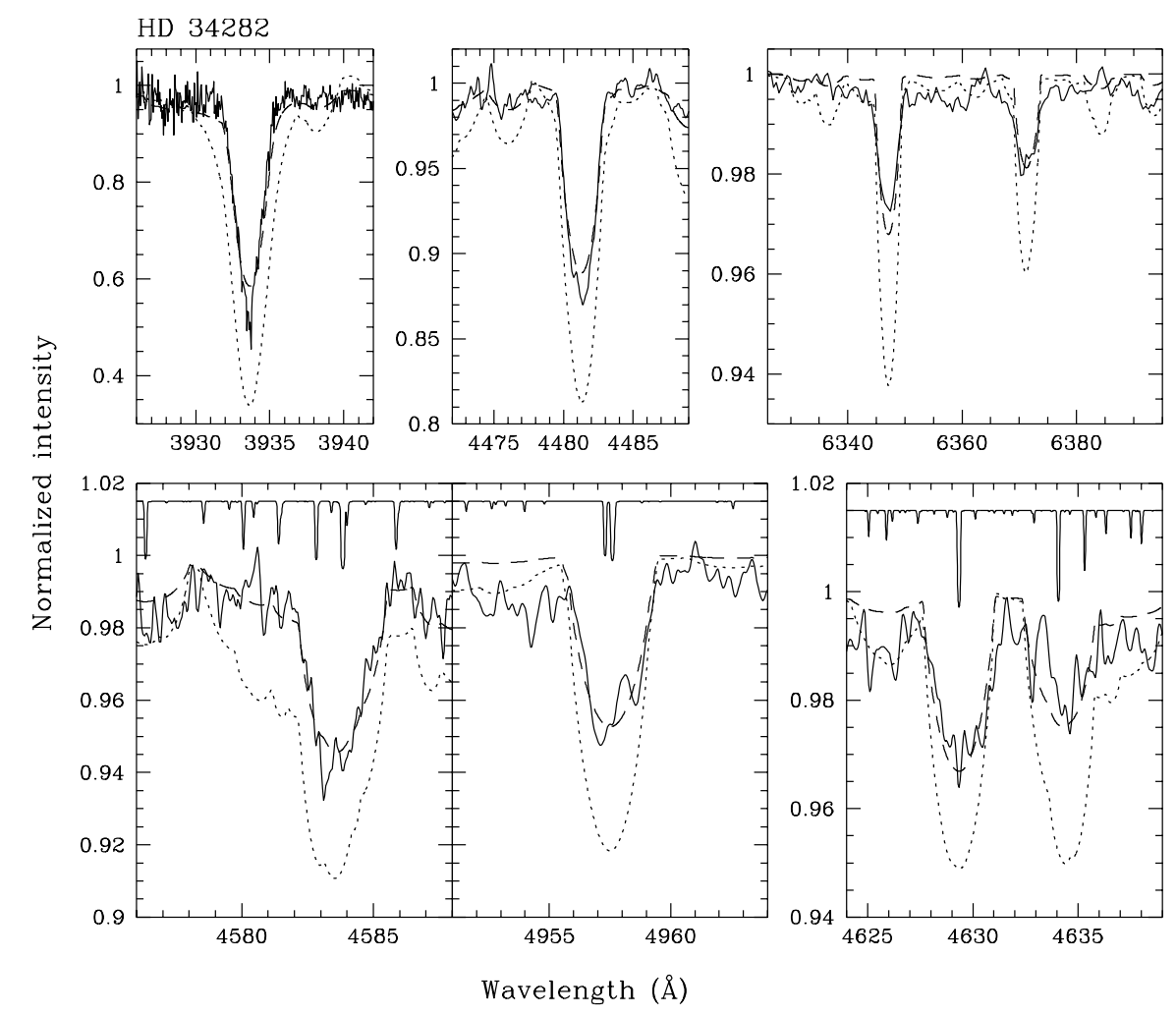

Fig. 5. Comparison of observed and synthetic spectral features of HD 34282. From top to bottom and left to right the following lines have

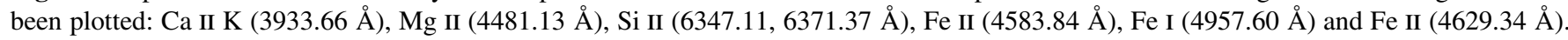
Observed spectra are plotted as solid lines. Synthetic spectra computed with $T_{\text {eff }}=8625 \mathrm{~K},[\mathrm{Fe} / \mathrm{H}]=-0.8$ and $\log g_{*}=4.20$ and broadened with $v \sin i=110 \mathrm{~km} \mathrm{~s}^{-1}$ are plotted as dashed lines. Dotted lines represent synthetic spectra computed with the same $T_{\text {eff }}, \log g_{*}$ and $v \sin i$ but using solar abundances. In the lower panels, the synthetic unbroadened spectra are shown to illustrate the lines giving rise to the observed profiles.

$4501.27 \AA$ (Ti II). For HD 141569 the projected rotational velocity is so high that it is difficult to find individual metallic lines; instead the regions 4260-4280 $\AA, 4375-4400 \AA$ and 4540-4560 $\AA$, which contain a large number of lines, have been selected. For both stars the lines $3933.66 \AA$ (Ca II), $4481.13 \AA$ (Mg II), and the Si II doublet at 6347.11, 6371.37 have also been used (the Si II doublet is clearly observed in the INT spectra). It is found, particularly in the case of HD 34282, that different $\left(T_{\text {eff }},[\mathrm{Fe} / \mathrm{H}]\right)$ pairs fit the observed spectra reasonably well. The comparison shows that higher effective temperatures require higher metallicities to fit a given spectral line. This $\left(T_{\text {eff }},[\mathrm{Fe} / \mathrm{H}]\right)$ degeneracy can be solved by using the observed SEDs, in particular the UBVRI photometry.

For each $\left(T_{\text {eff }},[\mathrm{Fe} / \mathrm{H}]\right)$ pair, which provides an apparent satisfactory fit to the observed spectra, we have computed the corresponding synthetic Kurucz SED in the spectral interval from 3000 to $10000 \AA$, i.e., the range from $U$ to $I$. The observed $U B V R I$ fluxes from each star have been dereddened at small steps of $E(B-V)$ and compared to the Kurucz synthetic models (we have covered the interval $0.0<E(B-V)<0.30$ with a stepsize of 0.01 ). The observed and synthetic photometry were normalized to $R$. It turns out that the overall SED shape is significantly more sensitive to the effective temperature than to the metallicity; thus, this method is particularly useful to pinpoint the best $T_{\text {eff }}$, and consequently the $\left(T_{\text {eff }},[\mathrm{Fe} / \mathrm{H}]\right)$ solution. The best $T_{\text {eff }}$ value fitting the data is chosen by means of a minimum $\chi^{2}$ criteria. Once the best values for $T_{\text {eff }}$ and $[\mathrm{Fe} / \mathrm{H}]$ have been found, the value of the gravity is re-calculated using the procedure described above with synthetic Balmer profiles corresponding to this metallicity. The process is iterated until convergence is achieved.

The optimum solutions found are (see also Table 4): $\mathrm{HD} 34282, T_{\text {eff }}=8625 \mathrm{~K},[\mathrm{Fe} / \mathrm{H}]=-0.8, \log g_{*}=4.20$; HD $141569, T_{\text {eff }}=10000 \mathrm{~K},[\mathrm{Fe} / \mathrm{H}]=-0.5, \log g_{*}=4.28$. The corresponding spectral types for these temperatures and gravities are $\mathrm{A} 3 \mathrm{~V}$ and $\mathrm{B} 9.5 \mathrm{~V}$ respectively. The relevant result is that both stars have low metal abundances. In addition, projected rotational velocities, $v \sin i$, of $110 \mathrm{~km} \mathrm{~s}^{-1}$ for HD 34282 and $236 \mathrm{~km} \mathrm{~s}^{-1}$ for HD 141569 provide a better agreement with the observed profiles than the $v \sin i$ values reported by Mora et al. (2001). Finally, the best $\chi^{2}$ fit gives $E(B-V)=0.05 \mathrm{mag}$ for HD 34282 and $0.12 \mathrm{mag}$ for HD 141569. We note that Mora et al. (2001) deduced $E(B-V)=0.15 \pm 0.10 \mathrm{mag}$ for the latter star from the graphite interstellar absorption band at $2200 \AA$ observed with the IUE, also Oudmaijer et al. (2001) found $A_{\mathrm{V}}=0.34 \pm 0.20$, which implies $E(B-V)=0.11 \pm 0.06$; both values of the extinction are in very good agreement with our present estimate. Figures 5 and 6 show the comparison between the observed and best synthetic spectra for several spectral lines and ranges; synthetic spectra using solar abundances are also plotted for comparison. Both figures clearly show that the observed spectra of HD 34282 and HD 141569 are much better reproduced with our low metallicity estimates and, therefore, a low metal content in the photospheres of both 


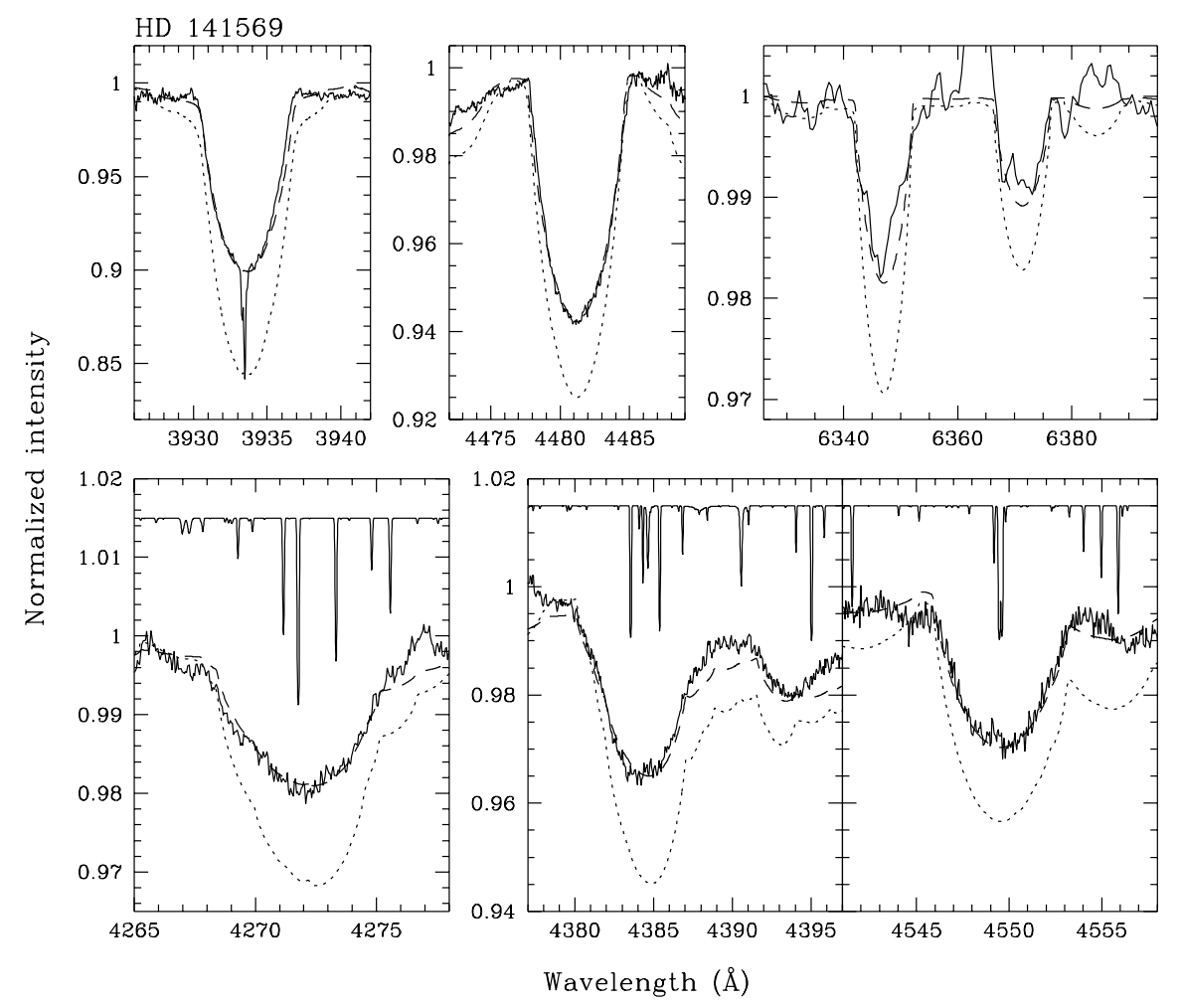

Fig. 6. Comparison of observed and synthetic spectral features of HD 141569. In the top panels, the spectral lines are the same as in Fig. 5. In the bottom panels, three spectral regions, instead of individual lines, are displayed. Observed spectra are plotted as solid lines. Synthetic spectra computed with $T_{\text {eff }}=10000 \mathrm{~K},[\mathrm{Fe} / \mathrm{H}]=-0.5$ and $\log g_{*}=4.28$ and broadened with $v \sin i=236 \mathrm{~km} \mathrm{~s}^{-1}$ are plotted as dashed lines. Dotted lines represent synthetic spectra computed with the same $T_{\text {eff }}, \log g_{*}$ and $v \sin i$ but using solar abundances. In the lower panels, the synthetic unbroadened spectra are shown to illustrate the lines giving rise to the observed profiles.

stars is strongly suggested. We note that HD 34282 has some similarities with HD 37411, another low metallicity PMS star (Gray \& Corbally 1998). With respect to HD 141569, Gray \& Corbally (1998) indicate that it is a "mildy metal-weak" star, in agreement with our results, though Dunkin et al. (1997) give a solar abundance.

\subsection{Stellar fluxes and infrared excesses. Luminosities, masses, ages and distances}

Once the stellar parameters defining the photospheres of HD 34282 and HD 141569 have been determined, we can calculate the stellar fluxes and infrared excesses. In addition, we can locate the stars in a HR diagram using appropriate evolutionary tracks and estimate their distances.

The stellar flux, $F_{*}$, is computed by integrating the best-fit Kurucz model normalized to the dereddened observed $R$-band flux, as given in Table 3. The infrared excess, $F_{\mathrm{IR}}$, is calculated by subtracting the flux under the tail of the bestfit Kurucz model from the observed fluxes, and integrating the result from $\lambda_{\mathrm{IR}}$, the wavelength where the SED separates from the tail of the Kurucz model (see Figs. 9 and 10). Extrapolations to long wavelengths following the formula by Chavarria et al. (1981) have been included in the integration. Table 4 shows the integrated fluxes for the stars and their IR excesses. HD 34282 has a $F_{\mathrm{IR}} / F_{*}$ ratio of $0.63 \pm 0.19$, which suggests that some intrinsic luminosity might be arising from the disk; $F_{\mathrm{IR}} / F_{*}$ values of $\sim 0.25$ and $\sim 0.5$ are expected from flat and flared optically thick reprocessing disks, respectively (Kenyon \& Hartmann 1987). In the case of HD 141569, we find $F_{\mathrm{IR}} / F_{*}=0.018 \pm 0.004$ and a passive disk with relatively low optical depth is suggested. This figure is one order of magnitude higher than typical values in Vega-type stars $\left(10^{-5}\right.$ to $10^{-3}$, Sylvester et al. 1996). We note that the ratio $F_{\mathrm{IR}} / F_{*}=$ $8 \times 10^{-3}$ given by Zuckerman et al. (1995) and usually quoted in the literature does not include the $1.35 \mathrm{~mm}$ flux measured by Sylvester et al. (2001). If we remove that flux from our estimates, we recover the figure given by Zuckerman et al.

Knowledge of the stellar metallicities is relevant since changes in the metal abundance, $Z$, produce non-negligible changes in the relative positions of the evolutionary tracks. In particular, a decrease in the metallicity moves one given track with a fixed value of the stellar mass, to lower luminosities, higher gravities and higher temperatures, apart from changing its overall shape. This effect is illustrated in Fig. 7 for the $\log g_{*}-\log T_{\text {eff }}$ HR diagram. The left two panels of Fig. 7 show the positions of the stars in the HR diagram according to the results found for $T_{\text {eff }}$ and $\log g_{*}$. The evolutionary tracks for a scaled solar mixture from the Yonsei-Yale group (Yi et al. 2001) - $Y^{2}$ in their notation - have been used in this work. The $\mathrm{Y}^{2}$ tracks with $Z=0.004$ and $Z=0.007$ are appropriate for HD 34282 and HD 141569, respectively. For comparison, 

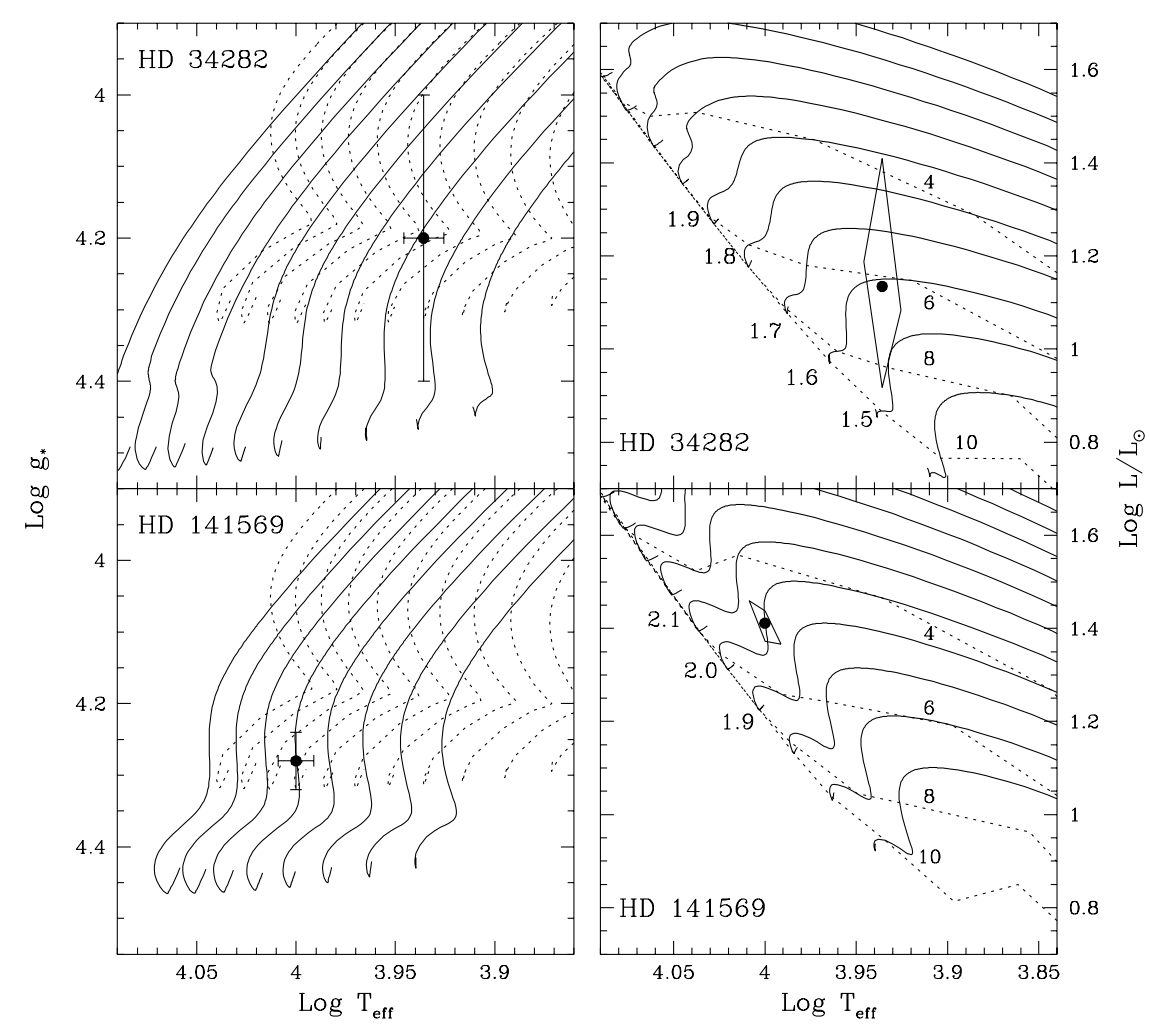

Fig. 7. Positions of the stars in the HR diagram according to the results shown in Table 4. Left. Upper panel: position of HD 34282 in the HR diagram $\log g_{*}-\log T_{\text {eff }}$. The $Y^{2}$ PMS tracks with $Z=0.004$ (solid lines) and $Z=0.02$ (solar, dotted lines) are plotted. Lower panel: same as in the upper panel for HD 141569. The tracks correspond to $Z=0.007$ (solid lines) and $Z=0.02$ (dotted lines). Right. Upper panel: position of HD 34282 in the HR diagram $\log L / L_{\odot}-\log T_{\text {eff. }}$ The PMS tracks are those for $Z=0.004$ (solid lines). Lower panel: same as in the upper panel for HD 141569. The tracks correspond to $Z=0.007$ (solid lines). In both cases we have labelled the tracks with the corresponding masses and the isochrones for 4, 6, 8 and $10 \mathrm{Myr}$ are shown with dotted lines.

the tracks with solar metallicity $(Z=0.02)$ are also included in the plot $^{3}$.

The corresponding values of the effective temperature and gravity are compared with the set of tracks and values for the luminosity, mass and age are derived. The position of HD 34282 and HD 141569 in the HR diagram $\log L / L_{\odot}-\log T_{\text {eff }}$ are plotted in the two panels at the right hand side of Fig. 7. The luminosities, combined with the total stellar fluxes $F_{*}$ obtained, imply distances of $348 \mathrm{pc}$ for HD 34282, and 108 pc for HD 141569. All the results are summarized in Table 4. The results for the luminosity, mass, age and distance are given in two columns for each star: in the first one the error bars derive from the uncertainties in $\log g_{*}$, keeping $T_{\text {eff }}$ fixed; in the second, the error bars derive from the uncertainties in $T_{\text {eff }}$, keeping $\log g_{*}$ fixed. The uncertainties for HD 34282 are larger than those for HD 141569 because of the larger uncertainties in the determination of the gravity. The vertices of the "diamond-like" boxes appearing in the two

\footnotetext{
${ }^{3}$ In the original $\mathrm{Y}^{2}$ set, there are no tracks for $Z=0.003$ and 0.006 , the appropriate values for each star considering that the relationship $\mathrm{Fe} / \mathrm{Fe}_{\odot}=X / X_{\odot}$ holds (in number if particles), where $X$ means any element with atomic number larger than 3 . The closest sets of tracks are those with $Z=0.004$ and 0.007 respectively. We have preferred to use original tracks kindly provided by the authors than interpolating between adjacent grids to build new sets of tracks and isochrones. In any case the differences are expected to be very small.
}

right-hand panels of Fig. 7 correspond to the tips of the error bars in the panels on the left.

Figure 7 shows that both stars lie above the main sequence in positions in agreement with their observational properties and current PMS evolution theory. The anomalous positions in the HR diagram previously reported for these stars referred to standard sets of tracks or photometric parameters computed with solar metallicity and a very doubtful distance in the case of HD 34282. For this star the anomalous position in the HR diagram is resolved both by the low metallicity and an appropriate distance estimate. In the case of HD 141569, its anomalous position originated only from the use of the wrong metallicity in the definition of the main sequence ${ }^{4}$.

The distance we obtain for HD $34282\left(348_{-77}^{+129} \mathrm{pc}\right)$ is comparable to that given by Piétu et al. $(2003)\left(400_{-100}^{+170} \mathrm{pc}\right)$. Both values are remarkably similar, and are more than $2 \sigma$ larger than the Hipparcos value. This leads us to consider that the Hipparcos parallax for this object has been overestimated. The stellar parameters quoted by Piétu et al. (2003) are compatible with ours, although we note their larger errors and their use of indirect methods to estimate the temperature and spectral type of the star; in addition, they use solar metallicity tracks which

\footnotetext{
${ }^{4}$ Note that in the two left-hand-side diagrams of Fig. 7 both stars also occupy correct positions in the HR diagram when compared with tracks with solar metallicity. If these tracks were used to obtain values of masses, luminosities and ages, these quantities would be incorrect.
} 
are not appropriate for this star. In the case of HD 141569 our distance is comparable to the Hipparcos one $\left(99_{-8}^{+9} \mathrm{pc}\right)$. We note that the age estimate by Weinberger et al. (2000) for the two nearby low mass stars is similar to that deduced by us for HD 141569 directly. This result reinforces the idea that the three stars are real companions.

Finally, estimates of the extinctions and distances can also be made using the absolute magnitudes and colours provided by the $\mathrm{Y}^{2}$ isochrones. Taking the values of mass, age and metallicity given in Table 4 and the observed values of $B$ and $V$ used to construct the SEDs (see Sect. 4.2 and Table 2), the extinctions $E(B-V)$ obtained are 0.04 for HD 34282 and 0.06 for HD 141569. The former value especially is in excellent agreement with that given by the $\chi^{2}$ analysis. The absolute magnitudes $M_{V}$ provided by the tracks are 2.04 and 1.37 respectively; these, combined with the observed values of $V$ corrected with the above extinctions, yield distances of $347 \mathrm{pc}$ for HD 34282 and $129 \mathrm{pc}$ for HD 141569, again in good agreement with the results shown in Table 4.

\section{Modelling the SEDs: The irradiated accretion disk models}

The origin of the IR excess in HAeBe stars has been a matter of intense debate (see e.g. Waters \& Waelkens 1998). Some authors argued in favor of models with an approximately spherical dusty envelope of low optical depth (Berrilli et al. 1992; di Francesco et al. 1994; Pezzuto et al. 1997; Miroshnichenko et al. 1997), others considered circumstellar disks (Hillenbrand et al. 1992; Chiang et al. 2001; Natta et al. 2001) or proposed the existence of both (Natta et al. 1993; Miroshnichenko et al. 1999). Ultimately the existence of disks around HAeBe stars was firmly established by direct imaging at millimetre wavelengths (Mannings et al. 1997; Mannings \& Sargent 1997, 2000; Testi et al. 2001; Piétu et al. 2003), in the optical (Grady et al. 1999, 2000) and in the IR (Close et al. 1997; Jayawardhana et al. 1998; Weinberger et al. 1999). Some authors have been able to fit the complete SEDs of HAeBe stars with passive irradiated disk models from Chiang \& Goldreich (Chiang et al. 2001; Natta et al. 2001).

\subsection{The models}

In this work we use the self-consistent irradiated accretion disk models from D'Alessio et al. $(1998,1999,2001)$ to fit the SEDs of HD 34282 and HD 141569. The methods used here were applied succesfully to fit the SEDs of young T Tauri stars with an approximate age of $1 \mathrm{Myr}$. This approach is valid for these two stars since they are young (see Sect. 5) and at least one of them seems to be still actively accreting. Since the calculations yield the vertical structure and emission properties of the disk self-consistently with the stellar parameters, and we have characterized the stellar photospheres with precision, a good fit to the SED would provide a physically based picture of the system without the use of ad hoc parametrizations for the disk temperature or surface density profiles.

The models involve the following assumptions: the disk is in a steady state $(\dot{M}=\mathrm{d} M / \mathrm{d} t$ is constant), it is geometrically thin $(H / R \ll 1$, where $H$ is the scale height of the disk and $R$ is the radial distance), the accretion viscosity is computed with $v=\alpha H c_{\mathrm{s}}$, following the $\alpha$-prescription from Shakura \& Sunyaev (1973), in which $c_{\mathrm{s}}$ is the sound speed. The dust and gas are well mixed in the whole disk with the usual dust to gas mass ratio of $1 / 100$. For the dust we use a grain size distribution $n(a)=n_{0} a^{-p}$ with $p$ values of 2.5 or 3.5 and a minimum grain size of $0.005 \mu \mathrm{m}$. Possible values for the maximum grain size are $1 \mu \mathrm{m}$ to $10 \mathrm{~cm}$. The abundances are those given by Pollack et al. (1994). The radiation field is considered in two separate regimes (one characteristic of the disk local temperature and one characteristic of the stellar effective temperature) as in Calvet et al. $(1991,1992)$ and finally the radiative transfer is done by solving the first two moments of the radiative transfer equation with the Eddington approximation (see D'Alessio et al. 1998, 1999, 2001 for further details).

The disk is the solution of the detailed vertical structure equations for each annulus, and the synthetic SEDs are obtained by solving the radiative transport equation in rays parallel to the line of sight and integrating (in solid angle) the disk's emission.

Note that, although the models we have applied are amongst the most sophisticated available, they have limitations inherent in the complexity of the physics and the computational techniques. For example, they assume a unique dust size distribution with a maximum grain size $a_{\max }$ and the same viscosity parameter $\alpha$ throughout, which in some real cases precludes fitting the observed SED with a single component. For HD 34282 a combination of two disk models is needed: one for the long wavelength part of the SED, with high viscosity and a maximum grain size of $1 \mathrm{~cm}$ (Sect. 6.2.2) and a second one for the mid-infrared region of the SED, with lower viscosity and a maximum grain size of $1 \mu \mathrm{m}$ (Sect. 6.2.3); the first one will be referred to as the mid-plane disk and the latter as the surface disk according to their vertical scale heights, of which the first has a lower value. We found that these two models were the only combination able to reproduce the whole SED of this star, implying that some vertical grain segregation may be present in the disk around HD 34282. In addition, to explain the bump at $3 \mu \mathrm{m}$ we propose the presence of a wall-like structure at the inner edge of the disk (Sect. 6.2.4). For HD 141569 a single disk model accounts for the whole SED (Sect. 6.3).

\subsection{The disk model for $H D 34282$}

The disk around HD 34282 shows signs of activity: optical and near-IR variability, $\mathrm{H} \alpha$ variable emission and high fractional IR excess. Hence, to fit the SED of HD 34282, we assume that the disk is heated by irradiation from the central star and viscous dissipation from the mass accretion onto the star.

For the central star the following stellar parameters are assumed (Table 4): $T_{\text {eff }}=8625 \mathrm{~K}, R_{*}=1.66 R_{\odot}, M_{*}=1.59 M_{\odot}$, $L_{*}=13.64 L_{\odot}$ and $d=348 \mathrm{pc}$, where the stellar radius was calculated from the stellar mass and the surface gravity. For the disk we take an inclination angle of $i=56^{\circ}$ (Piétu et al. 2003) and an outer radius of $705 \mathrm{AU}$, which is the disk radius given by these authors scaled from their distance of $400 \mathrm{pc}$ to our value of $348 \mathrm{pc}$. The inner disk radius is calculated 
self-consistently using the position of the near-IR bump, the result being $0.31 \mathrm{AU}$ (see Sect. 6.2.4).

To find the remaining input disk parameters we need to know the mass accretion rate towards the star (which will be derived from ultraviolet Walraven photometry), the disk viscosity $\alpha$ (which will be fitted to raise the appropriate flux at millimetre wavelengths once we know the mass accretion rate) and the dust properties (related to the slope in the millimetre range and the IR excess shape at shorter wavelengths).

\subsubsection{The mass accretion rate in the disk of HD 34282}

The fractional IR excess, $F_{\mathrm{IR}} / F_{*}$, of $\mathrm{HD} 34282$, namely $0.63 \pm 0.19$ (Table 4), is a little bit larger than the values measured in the SEDs of the HAeBe stars studied by Natta et al. (2001), all of them being smaller than 0.45 . These authors reproduce the SEDs assuming that no accretion is present, the reprocessing of radiation being the mechanism invoked to explain the IR excess. We propose that, given the more prominent IR excess of this star compared to those of the HAeBe stars in Natta et al. (2001) and the higher fractional IR excess (slightly larger than 0.5), accretion may be present in this object. For this reason, we first try to estimate the mass accretion rate towards HD 34282.

This parameter has been studied using Walraven ultraviolet photometry of HD 34282 (de Geus et al. 1990) and shock emission models by Muzerolle et al. (2003a), who apply to HAeBe stars the magnetospheric accretion scenario from Calvet \& Gullbring (1998) and Gullbring et al. (2000). This theory explains the UV excess emission seen in many T Tauri stars as arising from an accretion shock. In HAeBe stars, the emission from the shocked atmosphere modifies the underlying photospheric emission reducing the Balmer jump with respect to the normal main-sequence value in cases with high mass accretion rate and large filling factors.

Shock models for a central star with the HD 34282 stellar parameters were computed. The energy flux of the accretion column, $F$, is a free parameter that controls the SED emitted by the accretion shock and the total mass accretion rate is proportional to this energy flux and to the filling factor, $f$, for the accretion column (Muzerolle et al. 2003a).

Figure 8 shows the fit of the photospheric plus accretion flux (solid line) to the dereddened Walraven photometry. Synthetic Walraven fluxes have been estimated by convolving the total flux (Kurucz plus shock fluxes) with the Walraven passbands, and compared to the dereddened data. These synthetic points are plotted as squares in Fig. 8 and match the dereddened data (filled circles) so well that they are barely visible in the graph. In the inset we display an enlargement, in linear scales, of the region to the blue side of the Balmer jump to show the subtle difference between the stellar (dashed line) and stellar plus shock (solid line) fluxes.

We used the $\chi^{2}$-minimization method to check if some UV excess was present in HD 34282. For three typical values of the column energy fluxes (namely $1 \times 10^{11}, 3 \times 10^{11}$ and $1 \times 10^{12} \mathrm{erg} \mathrm{cm}^{-2} \mathrm{~s}^{-1}$ ) we added the accretion flux to the Kurucz model with different filling factors $f$ and performed the

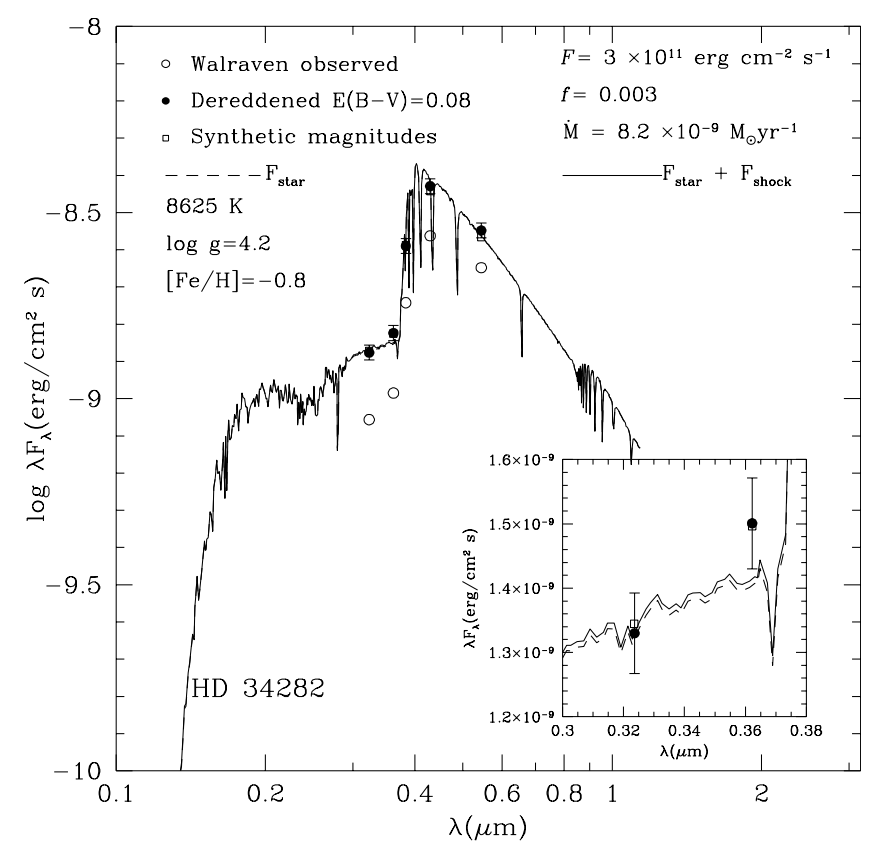

Fig. 8. Best-fit shock model fitting to the Walraven photometry of HD 34282. The open and filled circles are observed and dereddened Walraven photometry, respectively. The open squares are synthetic photometry computed with the total (stellar plus shock) flux. The solid line is the Kurucz model plus the accretion shock flux for the accretion column energy flux $F$ and filling factors $f$ specified in the plot. Shifted a small amount below this line would lie the Kurucz model for the stellar parameters of the star. The magnitude of this tiny difference can be seen in the inset where the Kurucz model has been plotted with a dashed line. The photometric point at $0.36 \mu \mathrm{m}$ lies above the models because the Walraven $B$ filter includes fluxes on both sides of the Balmer jump, hence the average is higher than the local flux at the blue side of the jump. The agreement between the dereddened and synthetic photometry is compatible with the presence of a weak accretion shock signature in the ultraviolet colours of the star.

$\chi^{2}$ estimation for the fittings. Since the Walraven photometry was not contemporaneous with the EXPORT observations, the best-fit value for $E(B-V)$ was 0.08 in this case. The quality of the fits to the data is very good, but, as the observational errors are much larger than the difference between models with and without accretion flux, we can not distinguish between these models. This happened for all three values of the column energy flux used and in all cases the mass accretion rate derived was very similar, the average value being $\dot{M}=(8.2 \pm 0.2) \times 10^{-9} M_{\odot} \mathrm{yr}^{-1}$. This value is consistent with the low accretion rates expected in HAeBe stars (Hartmann et al. 1993; Ghandour et al. 1994; Natta et al. 2001) and also with estimates for UX Ori-like stars (Tambovtseva \& Grinin 2000).

Given the tiny difference introduced by the shock and the small $\chi^{2}$ values obtained, it is clear that the excess flux is virtually impossible to measure in this case and that this determination constrains the mass accretion rate mostly as an upper limit. Once this method has been applied to other HAeBe stars with different amounts of IR excess, we will have better knowledge of its reliability. However, here we will adopt this value for the mass accretion rate toward the star because, being compatible with the ultraviolet photometry, it is also consistent with 


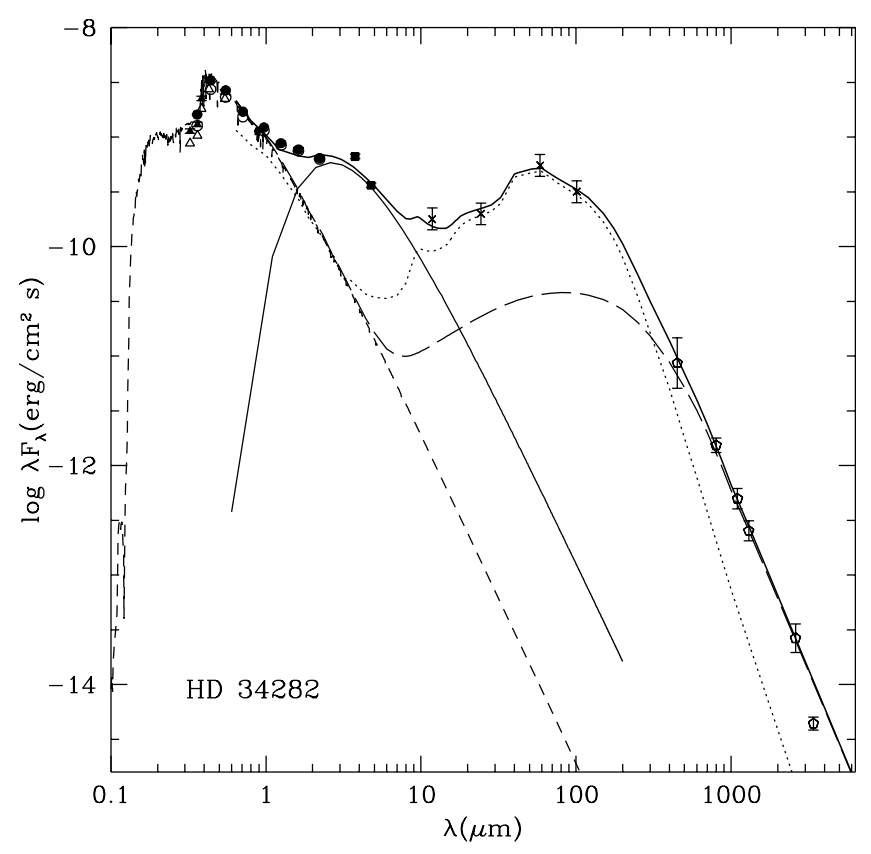

Fig. 9. Disk models fitting the full SED of HD 34282. Filled triangles are dereddened Walraven photometry from de Geus et al. (1990). Filled circles are dereddened EXPORT optical and near-IR photometry. Filled squares are $L M$ magnitudes from Sylvester et al. (1996). Open symbols are the observed fluxes. The crosses are the IRAS colour-corrected fluxes and the open pentagons are submillimetre fluxes from Sylvester et al. (1996), Piétu et al. (2003) and Mannings \& Sargent (2000). The dashed line is the Kurucz model for the stellar parameters computed in Sect. 5. The thin solid line is the $1400 \mathrm{~K}$ black body fit to the near-IR excess. The dotted line is the contribution of the surface model with inclination angle $i=56^{\circ}$, $\dot{M}=8.2 \times 10^{-9} M_{\odot} \mathrm{yr}^{-1}, \alpha=0.008, R_{\text {in }}=0.31 \mathrm{AU}, R_{\text {out }}=705 \mathrm{AU}$, $p=3.5$ and $a_{\max }=1 \mu \mathrm{m}$. The long-dashed line is the contribution of the mid-plane disk model with the same parameters as the surface one except $p=2.5, a_{\max }=1 \mathrm{~cm}$ and $\alpha=0.0008$. The thick solid line on the top of the others is the addition of all components. See the text for explanation.

the physical parameters needed to fit the SED millimetre fluxes (see Sect. 6.2.2). In the next three subsections we describe in detail how three different components account for the observed SED of HD 34282.

\subsubsection{The long wavelength part of the SED}

The flux at $1.3 \mathrm{~mm}$ is proportional to the mass of the disk since its thermal emission is nearly optically thin at this wavelength (see e.g., Mannings 1994; Natta et al. 1997). Therefore we can use the flux density at $1.3 \mathrm{~mm}$ (Piétu et al. 2003) to estimate the viscosity parameter $\alpha$ of the system. Under the assumption of steady accretion, the disk surface density scales as $\Sigma \propto \dot{M} \alpha^{-1} T^{-1}, \Sigma$ being the surface density, $\dot{M}$ the mass accretion rate, $\alpha$ the viscosity parameter, and $T$ the temperature of the mid-plane disk (D'Alessio et al. 1999). For moderate accretion rates, the heating of the disk is dominated by stellar irradiation and $T$ will not change very much with the mass accretion rate, so the mass of the disk will be roughly constant for constant $\dot{M} \alpha^{-1}$. Usual values of $\alpha$ range from 0.1 to $0.001,0.01$ being the one that reproduces best the millimetre fluxes in T Tauri

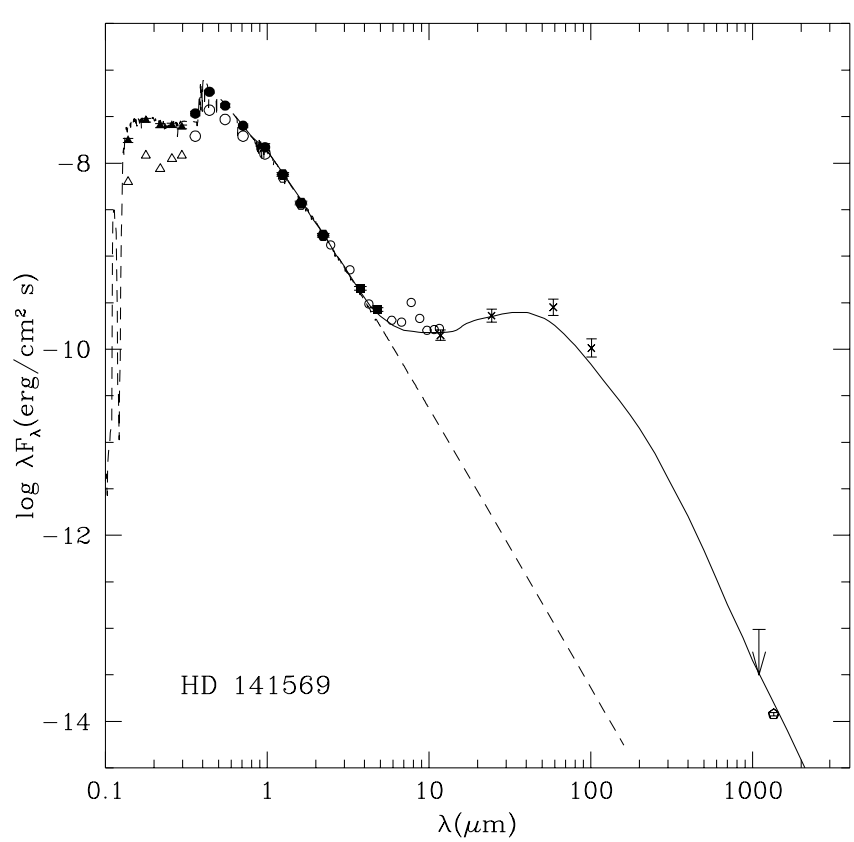

Fig. 10. Disk model fitting the SED of HD 141569. Filled triangles are dereddened IUE data. Filled circles are dereddened EXPORT optical magnitudes. Filled hexagons are dereddened $J H K$ magnitudes from Malfait et al. (1998) and filled squares are $L M$ magnitudes from Sylvester et al. (1996). The corresponding open symbols are the observed fluxes. The small open circles are the ISO-PHOT measurements. The crosses are the IRAS colour-corrected fluxes, the open pentagon is the submillimetre flux from Sylvester et al. (2001) and the arrow is the upper limit flux from Sylvester et al. (1996). The dashed line is the Kurucz model for the stellar parameters computed in Sect. 5. The thick solid line is the disk model with inclination angle $i=51^{\circ}$, $\dot{M}=10^{-11} M_{\odot} \mathrm{yr}^{-1}, \alpha=0.001, R_{\text {in }}=0.24 \mathrm{AU}, R_{\text {out }}=428 \mathrm{AU}$, $p=2.5$ and $a_{\max }=1 \mathrm{~mm}$. See text for details.

stars (D'Alessio et al. 2001). We find that $\alpha=0.0008$ yields the appropriate millimetre flux for HD 34282, given the stellar and disk parameters considered.

The slope of the SED at millimetre wavelengths $\alpha_{\mathrm{mm}}$ is normally modelled with the $\beta$ dependence of the opacity $\kappa_{\lambda}=$ $\kappa_{0}\left(\lambda / \lambda_{0}\right)^{-\beta}$ (Beckwith et al. 1990; Beckwith \& Sargent 1991) where $\alpha_{\mathrm{mm}}=\beta+2$ to account for the growth of the dust grains. In our case, the millimetre slope is controlled by the dust distribution and the optical depth of the disk. To obtain the observed millimetre slope $\alpha_{\mathrm{mm}}=2.2\left(F_{v} \propto v^{2.2}\right)$, a combination of $p=2.5$ and a maximum grain size $a_{\max }=1 \mathrm{~cm}$ was needed. This value of $p$ can be interpreted as the resulting size distribution of a coagulation process in which most of the disk dust mass resides in large grains (Miyake \& Nakagawa 1993).

The contribution of the mid-plane disk to the SED is shown in Fig. 9 as a long-dashed line. There is an excess flux with respect to the observed flux at $3.4 \mathrm{~mm}$ measured by Piétu et al. (2003). We consider the sub-millimetre and millimetre slope well fitted with $F_{v} \propto v^{2.2}$ since five flux densities, between 0.45 and $2.6 \mathrm{~mm}$, are consistent with it, and no significant change in the slope is expected (see, D'Alessio et al. 1990, but also Qi et al. 2003 for a counter-example). Missing flux, a sudden change in the dust opacity at large wavelengths or the contribution of optically thick regions at small radii may explain the 
Table 5. Disk model parameters and results.

\begin{tabular}{lccc}
\hline \hline \multicolumn{3}{c}{ HD 34282 } & HD 141569 \\
\hline$\dot{4}$ Mid-plane & Surface \\
\hline$\dot{M}\left(M_{\odot} \mathrm{yr}^{-1}\right)$ & $8 \times 10^{-9}$ & $8 \times 10^{-9}$ & $1 \times 10^{-11}$ \\
$\alpha$ & $8 \times 10^{-4}$ & $8 \times 10^{-3}$ & $1 \times 10^{-3}$ \\
$R_{\text {in }}(\mathrm{AU})$ & 0.31 & 0.31 & 0.24 \\
$R_{\text {out }}(\mathrm{AU})$ & 705 & 705 & 428 \\
$p$ & 2.5 & 3.5 & 2.5 \\
$a_{\max }$ & $1 \mathrm{~cm}$ & $1 \mu \mathrm{m}$ & $1 \mathrm{~mm}$ \\
Inclination & $56^{\circ}$ & $56^{\circ}$ & $51^{\circ}$ \\
\hline$H_{10 \mathrm{AU}}(\mathrm{AU})$ & 3.06 & 4.13 & 2.33 \\
$M_{\text {disk }}\left(M_{\odot}\right)$ & 0.70 & 0.06 & $6.3 \times 10^{-4}$ \\
\hline
\end{tabular}

discrepancy (e.g. D'Alessio et al. 2003). A way to check the validity of this result would be to simulate the observation of this model for the mid-plane disk in the same line observed by Piétu et al. (2003), namely the ${ }^{12} \mathrm{CO} J=2 \rightarrow 1$ transition, and compare the resulting map with the observations. This work is being done and it will be presented elsewhere.

\subsubsection{The mid-infrared region of the SED}

As can be seen in Fig. 9 the IR emission coming from a single irradiated accretion disk model does not reproduce the observed mid-IR fluxes. According to the CIRR values in the IRAS PSC, the cirrus infrared emission around this star is not an important contribution to the measured fluxes at 60 and $100 \mu \mathrm{m}$, so we consider that the flux in this part of the SED is also coming from the disk, implying that a second component is required.

The IR excess produced by an irradiated accretion disk model with dust and gas well mixed decreases as the size of the dust particles increases (D'Alessio et al. 2001). For a fixed dust mass, a decrease in the amount of large dust particles obviously implies the presence of more small grains in the disk; the effect is that the opacity of the dust to the stellar radiation increases and the height where most of the stellar radiation is deposited is greater. In that case, the disk intercepts more radiation from the star and hence it will emit more IR flux. In order to fit the mid-IR excess in HD 34282, we need the contribution of a disk component that we will call the surface disk with $a_{\max }=1 \mu \mathrm{m}, p=3.5$, the same mass accretion rate as the mid-plane disk (in this way we assure that the mass transfer is conserved through the disk) and $\alpha=0.008$. The contribution of the surface disk to the SED is shown in Fig. 9 as a dotted line.

Considering both components, the disk would contain large grains in the mid-plane, which are responsible for the almost optically thin emission at sub-millimetre and millimetre wavelengths and a population of small grains - the surface disk - on top of the previous model, which is responsible for the emission in the mid-IR. The total mass of the disk around HD 34282 can now be estimated from the masses of both disk components (Table 5) assuming that the mid-plane disk extends out to some intermediate height and the surface disk occupies the higher layer to the end of the disk. Given that the disks are in hydrostatic equilibrium, the density distribution with height is a Gaussian function centered at the mid-plane. The total mass is then dominated by the mid-plane disk and lies between 0.6 and $0.7 M_{\odot}$. The disk mass is less than half the stellar mass, supporting the hypothesis that the main gravitational field is due to the central star.

Natta et al. (2000) used the flux at 2.6 millimetres to estimate a mass of $0.05 M_{\odot}$ for the disk of HD 34282, an order of magnitude smaller than our value. Differences in the gas temperature and the opacity parametrization used in their calculation may account for the difference. Nevertheless, the upper limit of the disk masses computed by Natta and collaborators for a large sample of $\mathrm{HAeBe}$ stars matches our estimate for the mass of the disk around HD 34282, showing that it is quite massive compared to typical HAeBe disks.

\subsubsection{The near-IR wavelength region of the disk}

Some HAeBe stars show conspicuous near-IR bumps. Hillenbrand et al. (1992) used high mass accretion rate models to fit their SEDs but Hartmann et al. (1993) argued that such large values for $\dot{M}$ were incompatible with the bolometric luminosity of the objects. Finally Natta et al. (2001) proposed the presence of a frontally illuminated wall at the dust destruction radius where this near-IR bump would be emitted making compatible the shape of the SED and the luminosity of the object.

We assume that the excess emission at $3 \mu \mathrm{m}$ in the SED of HD 34282 originates in a wall-like structure at the inner part of the disk and model it as a black body at $1400 \mathrm{~K}$. Following Natta et al. (2001) and Dullemond et al. (2001), if this wall of irradiated material were optically thick then it would radiate as a black body at the local temperature, namely that of dust destruction (according to Pollack et al. 1994, $T_{\text {evap }} \sim$ 1200-1400 K).

The position, $R_{\text {rim }}$, and height, $H_{\text {rim }}$, of this irradiated wall can be computed following the methods described in Dullemond et al. (2001). Also, Muzerolle et al. (2003b) have recently fitted the near-IR bump in the SEDs of Classical $\mathrm{T}$ Tauri stars with a black body arising from a similar model for a dust destruction wall. These authors find best-fit temperatures for the wall to be around $1400 \mathrm{~K}$. The main difference with respect to the first model is that the dust is not treated as optically thick but the position of the rim is computed with the same dust size distributions as in D'Alessio et al. (2001) and the methods used in Calvet et al. (1991, 1992).

The projected area, $A$, of the rim is computed as in Appendix B in Dullemond et al. (2001). The solid angle subtended by this area is $A / d^{2}$, where $d$ is the distance to the source. The normalization constant needed to fit a black body to the near-IR photometry is proportional to the solid angle subtended by the rim, so after computing the position of the wall, $R_{\text {rim }}$, we can estimate the corresponding height so that the emitting area is consistent with the photometry.

Then, using Eq. (14) in Dullemond et al. (2001) in a simple iterative procedure, we get $R_{\text {rim }}=0.31 \mathrm{AU}$ and $H_{\text {rim }}=$ $0.06 \mathrm{AU}$. Also, using the equation for $R_{\text {rim }}$ in Muzerolle et al. (2003b) similar results were obtained (namely $R_{\text {rim }}=0.31 \mathrm{AU}$ 
and $\left.H_{\text {rim }}=0.04 \mathrm{AU}\right)$. This agreement is due to the fact that the different optical depth assumed for the dust in Muzerolle et al. (2003) is compensated by the autoirradiation correction introduced by Dullemond et al. (2001).

It is remarkable that the height obtained in this way is similar to the height of the surface disk component at $R_{\text {rim }}$ $\left(H_{\text {model }}=0.06 \mathrm{AU}\right)$. However, we must take into account that the height of a single disk where the large grains are in the mid-plane and the small grains in upper layers may be different to this ${ }^{5}$. Firmer conclusions about these heights require a selfconsistent determination of the vertical structure with settled dust, which is left for future work.

As quoted in Natta et al. (2001), the hypothesis of the irradiated wall assumes a negligible opacity of the gas disk inside $R_{\text {rim }}$ so that the stellar radiation is able to reach the inner wall. The calculation of the actual gas opacity inside the wall is difficult because of the multiple atomic and molecular absorption lines present. Muzerolle et al. (2003b) studied the opacity of a gaseous disk inside the dust destruction radius using an extended set of gas opacities and a detailed treatment of the heating of the gas due to accretion and stellar irradiation. They found that for typical parameters of HAeBe stars, the optical depth of the inner hole would be low enough to allow a substantial fraction of the stellar radiation to reach the dust sublimation wall.

The thin solid line in Fig. 9 is the contribution of the irradiated wall to the SED. The thick solid line is the addition of the Kurucz model and the contributions to the IR excess of the wall and the mid-plane and surface disks. In Table 5 a summary of the input parameters for the models is given. Also the values found for the height at $10 \mathrm{AU}$ and the disks' masses for each component are given.

\subsection{The disk model for HD 141569}

The SED of this star has already been fitted with optically thin dust models (e.g. Sylvester et al. 1996; Malfait et al. 1998). Recently Li \& Lunine (2003) have published a highly detailed, thorough model in which the star is taken to be a Vega-type object with a debris disk filled with optically thin porous dust. We have shown in previous sections, however, that HD 141569 is a PMS HAeBe star and not a Vega-type MS star, surrounded by a CS disk. This disk is clearly more evolved than usual CS disks detected around other HAeBe stars, but it cannot be considered as a "typical" debris disk, as indicated by the ratio between the IR excess and the bolometric luminosity and the non-negligible gas content and activity. Thus, the HD 141569 disk appears to be in an intermediate stage between "first generation" PMS disks and "second generation" debris disks. We take this as a justification to carry out an exploratory study in which we fit the SED of HD 141569 with PMS disk models and then compare the results with those from the debris disks models,

\footnotetext{
5 This is a consequence of the fact that the model with large $a_{\max }$ has a smaller vertical scale height - is less flared - than the model with small $a_{\max }$ (see Table 5), so the dust in the composite model would look like a slighly flared distribution of large particles surrounded by a more flared structure of smaller particles.
}

looking for convergent aspects. Note also that A-type Vegalike stars with debris disks are older systems than HD 141569 (Song et al. 2001).

The observational results strongly indicate that there is no significant mass accretion. Thus, we assume that the disk is heated only by irradiation from the central star and that no viscous dissipation from the mass accretion onto the star is present, which translates in the models we are using into an extremely low mass accretion rate. The central star has the following parameters (Sect. 5): $T_{\text {eff }}=10000 \mathrm{~K}, R_{*}=1.70 R_{\odot}$, $M_{*}=2.00 M_{\odot}, L_{*}=25.77 L_{\odot}$ and $d=108 \mathrm{pc}$, where the stellar radius was calculated from the stellar mass and the surface gravity. For the disk, we will use an inclination angle $i=51^{\circ}$ (Weinberger et al. 1999) and an outer radius $R_{\text {out }}=428 \mathrm{AU}$, i.e. the value of 400 AU given by Weinberger et al. (1999) scaled to our estimated distance of $108 \mathrm{pc}$. A change in the outer radius of no more than 50-60 AU will not affect substantially the final results. The inner radius is fitted to be $R_{\text {in }}=0.24 \mathrm{AU}$ to account for the lack of near-IR excess in the SED.

Figure 10 shows the results of our irradiated disk model compared to the SED of HD 141569. The peak at $7.7 \mu \mathrm{m}$ is a PAH feature (see Li \& Lunine 2003) that has not been modelled because it is not included in the dust model used (D'Alessio et al. 2001; Pollack et al. 1994). We find that a disk with a negligible mass accretion rate, $\dot{M} \sim 10^{-11} M_{\odot} \mathrm{yr}^{-1}$, and $\alpha=0.001$ (which translates into a dust and gas distribution of $\Sigma \propto R^{-1.0}$ with $\left.\Sigma(10 \mathrm{AU})=3 \mathrm{~g} \mathrm{~cm}^{-2}\right)$, and a dust distribution with $a_{\max }=1 \mathrm{~mm}$ and $p=2.5$ fits reasonably well the multiwavelength SED of HD 141569. The fractional IR luminosity from our model is 0.011 , consistent with the measured value (Sect. 5). The total mass (gas and dust) of our disk is $6.4 \times 10^{-4} M_{\odot}$ which is equivalent to $213 M_{\oplus}$. The mass of the disk derived with this model is higher than the typical masses encountered in Vega-like disks (Thi et al. 2001) but clearly smaller than those found in HAeBe stars (Natta et al. 1997), as expected given the evolutionary status of HD 141569. Table 5 gives a summary of the input parameters for the model as well as the mass of the disk and the height at $10 \mathrm{AU}^{6}$.

In the model by Li \& Lunine (2003) the surface density of dust particles is introduced with three analytical approximations that reproduce the surface brightness profiles observed in thermal and scattered light images by Augereau et al. (1999), Weinberger et al. (1999), Mouillet et al. (2001) and Marsh et al. (2002). However, the vertical height of the dust is computed assuming vertical hydrostatic equilibrium with a gas temperature taken to be a power law of the distance from the central star. In our disk models, both the density profile and the vertical scale height are computed taking into account the stellar irradiation heating (assuming that dust and gas are in thermal equilibrium). Concerning the continuum emission in the infrared to

\footnotetext{
6 Note that, whereas the disk of HD 141569 seems to be more evolved than that of HD 34282, the estimated age of HD 141569 is less than that of HD 34282 (see Table 4). However, one has to be careful when comparing this parameter for both stars, given the fact that HD 141569 is more massive and has larger metal abundance than HD 34282. Therefore, the evolutionary time scales are different for both objects. The initial conditions of the clouds where the stars and disks formed must also play a role in the evolutionary scenario.
} 
millimetre wavelengths, the presence of gaps of moderate width with smaller dust density does not change dramatically the shape of the SED (see e.g. Fig. 3 in Li \& Lunine 2003 where the three dust components are added to yield the smooth observed SED). Therefore we can assume that our continuous dust distribution is a good first approximation to the actual dust density with the advantage that it is self-consistently computed, along with its vertical hydrostatic structure, with the set of stellar parameters previously determined. This is in agreement with the results obtained by Clampin et al. (2003).

Concerning the dust, Li \& Lunine (2003) found that a "cold-coagulation" dust composed of porous dust grains with a porosity $P=0.9$ (90\% of the volume is vacuum and $10 \%$ material), and a power law distribution of sizes $n(a) \propto a^{-3.3}$ with $1 \mu \mathrm{m}<a<1 \mathrm{~cm}$, fitted very accurately the whole continuum SED of HD 141569. This dust model is not very far from our assumed $n(a) \propto a^{-2.5}$ with $0.005 \mu \mathrm{m}<a<1 \mathrm{~mm}$ mostly at large sizes. The difference in the optical properties of the compact dust grains compared to the porous dust grains may be accounted for by using a different power law exponent for the size distribution. The contribution of the PAH molecules to the IR emission is more important around the peaks at 3.3, 6.6, 7.7, $11.3 \mu \mathrm{m}$ (see Li \& Draine 2001 for details) and the continuum is well reproduced by the porous or compact dust populations.

The main effect of having a disk with gas and dust, instead of a pure dusty disk, is that the heating due to stellar irradiation produces an increase in the vertical scale height of the disk, yielding a more dilute dust population. Summarizing, we find that our approach fits the observed SED of HD 141569 reasonably well, although the model we have used does not include as many details as that developed by Li \& Lunine, particulary in the treatment of the dust properties. In this sense, our result is interesting since it shows the possibility of studying CS disks in transition objects from both perspectives, namely the PMS and MS sides.

\section{Conclusions}

We have presented a study of the properties and spectral energy distributions of the HAeBe stars HD 34282 and HD 141569 and their disks, based mainly on observations made by the EXPORT consortium (Eiroa et al. 2000). The main conclusions can be summarized as follows:

- The spectroscopic and photometric observations of both stars have been described and analysed in detail with the aim of throwing light on their evolutionary status and the dynamics and variability of their photospheres and disks.

- Both stars are metal-deficient. Our estimates are $[\mathrm{Fe} / \mathrm{H}]=-0.8$ for HD 34282 and -0.5 for HD 141569 .

- The analysis has provided values for the stellar distances. The distance to HD 34282 , namely 348 pc, is consistent with the value given by Piétu et al. (2003) and corrects the Hipparcos distance that is uncertain due to the large error in the parallax. The new distance for HD 34282, together with its low metallicity, resolves the anomalous position of this star in the HR diagram previously reported under the assumption that the star has solar abundances.
The distance to HD 141569 , namely $108 \mathrm{pc}$, is consistent with the Hipparcos data and in this case, the question of the anomalous position of this star in the HR diagram is resolved by using the correct set of tracks with the appropriate metallicity.

- In addition to the above two parameters, our analysis has provided for both stars values for the effective temperatures, spectral types, masses, gravities, luminosities, ages, projected rotational velocities and extinctions. These results are given in Table 4.

- Complete SEDs from the ultraviolet to the millimetre range have been constructed for both stars and their disks using the EXPORT data plus additional results gathered from the literature and on-line databases. Table 3 gives a full account of this compilation and the data sources.

- The self-consistent irradiated accretion disk models of D'Alessio et al. $(1998,1999,2001)$ have been used to fit the SEDs of both systems. A summary of the disk parameters obtained is given in Table 5.

- The SED of the disk of HD 34282 has been reproduced using a three-component model. The mid-plane disk is modelled with a maximum dust grain size of $1 \mathrm{~cm}$ and emits mainly at submillimetre and millimetre wavelengths, the surface disk model has a maximum grain size of $1 \mu \mathrm{m}$ and is responsible for the mid-IR excess emission. A near-IR bump similar to those seen in other HAeBe stars (see Natta et al. 2001) is fitted with the emission of a frontally illuminated wall placed at the dust destruction radius (Dullemond et al. 2001; Muzerolle et al. 2003b; D’ Alessio et al. 2003). The geometry of the wall is constrained by the near-IR photometry.

- In the process of fitting the SED of HD 34282, we have tried for the first time a new method to estimate the mass accretion rate towards a $\mathrm{HAeBe}$ star using ultraviolet Walraven photometry and the magnetospheric accretion shock models of Muzerolle et al. (2003a). We obtained un upper limit for the mass accretion rate in HD 34282 compatible with the parameters needed to fit the millimetre SED. The low value found does not support the idea by Herbst \& Shevchenko (1999) about the FU Ori-like mechanism causing the photometric activity of UX Ori stars.

- The SED of HD 141569 has been fitted with a disk model irradiated by the central star with negligible accretion and with large grains $\left(a_{\max }=1 \mathrm{~mm}\right)$. A slightly flared dust and gas distribution was obtained, which is consistent with a substantial amount of residual gas in the disk.

Acknowledgements. We would like to acknowledge Prof. Vladimir Grinin for his careful reading of the manuscript and his constructive suggestions. The authors are also grateful to Sukyoung Yi for kindly providing unpublished smoothed evolutionary tracks and for advice on their use, and to David Barrado y Navascués, Pedro García-Lario, José Francisco Gómez and Pedro Rodríguez for discussions. The work of C. Eiroa, B. Merín, B. Montesinos, A. Mora and E. Solano has been supported in part by the Spanish grant AYA2001-1124-C02. P. D'Alessio acknowledges grants from DGAPA-UNAM and CONACyT, México. B. Merín 
wishes to acknowledge the hospitality of the Young Stars' group at the Harvard-Smithsonian Center for Astrophysics and its support with the STScI grant GO-9524 during his stay as a visiting student, and to the INTA for its financial support of a graduate fellowship.

\section{References}

Alvarez, M., \& Schuster, W. J. 1981, RMxA\&A, 6, 163

Andrillat, Y., Jaschek, M., \& Jaschek, C. 1990, A\&A, 233, 474

Augereau, J. C., Lagrange, A. M., Mouillet, D., \& Ménard, F. 1999, A\&A, 350, L51

Beckwith, S. V. W., \& Sargent, A. I. 1991, ApJ, 381, 250

Beckwith, S. V. W., Sargent, A. I., Chini, R. S., \& Guesten, R. 1990, AJ, 99, 924

Berrilli, F., Corciulo, G., Ingrosso, G., et al. 1992, ApJ, 398, 254

Boccaletti, A., Augereau, J. C., Marchis, F., \& Hahn, J. 2003, ApJ, 585,494

Böhm, T., \& Catala, C. 1994, A\&A, 290, 167

Böhm, T., \& Catala, C. 1995, A\&A, 301, 155

Bouret, J.-C., \& Catala, C. 2000, A\&A, 359, 1011

Brittain, S. D., \& Rettig, T. W. 2002, Nature, 418, 57

Brittain, S. D., Rettig, T. W., Simon, Th., et al. 2003, ApJ, 588, 535

Calvet, N., \& Gullbring, E. 1998, ApJ, 509, 802

Calvet, N., Patino, A., Magris, G. C., \& D’Alessio, P. 1991, ApJ, 380, 617

Calvet, N., Magris, G. C., Patino, A., \& D’Alessio, P. 1992, RMxA\&A, 24, 27

Chavarría, C. 1981, A\&A, 101, 105

Chiang, E. I., \& Goldreich, P. 1997, ApJ, 490, 368

Chiang, E. I., \& Goldreich, P. 1999, ApJ, 519, 279

Chiang, E. I., Joung, M. K., Creech-Eakman, M. J., et al. 2001, ApJ, 547, 1077

Clampin, M., Krist, J. E., Ardila, D. R., et al. 2003, AJ, 126, 385

Close, L. M., Roddier, F., Hora, J. L., et al. 1997, ApJ, 489, 210

Corcoran, M., \& Ray, T. P. 1997, A\&A, 321, 189

Coulson, I. M., Walther, D. M., \& Dent, W. R. F. 1998, MNRAS, 296, 934

D’Alessio, P., Cantó, J., Calvet, N., \& Lizano, S. 1998, ApJ, 500, 411

D’Alessio, P., Calvet, N., Hartmann, L., Lizano, S., \& Cantó, J. 1999, ApJ, 527, 893

D’Alessio, P., Calvet, N., \& Hartmann, L. 2001, ApJ, 553, 321

D'Alessio, P., Calvet, N., Hartmann, L., et al. 2003, ASP Conf. Ser., 221, submitted

de Geus, E. J., Lub, J., \& van de Grift, E. 1990, A\&AS, 85, 915

de Winter, D., van den Ancker, M. E., Maira, A., et al. 2001, A\&A, 380,609

di Francesco, J., Evans, N. J., II, Harvey, P. M., Mundy, L. G., \& Butner, H. M. 1994, ApJ, 432, 710

Dullemond, C. P., Dominik, C., \& Natta, A. 2001, ApJ, 560, 957

Dunkin, S. K., Barlow, M. J., \& Ryan, S. G. 1997, MNRAS, 286, 604

Eiroa, C., Mora, A., Palacios, J., et al. (EXPORT) 2000, in Disks, Planetesimals and Planets, ed. F. Garzón, C. Eiroa, D. de Winter, $\&$ T. Mahoney, ASP Conf. Ser., 219, 3

Eiroa, C., Garzón, F., Alberdi, A., et al. (EXPORT) 2001, A\&A, 365, 110

Eiroa, C., Oudmaijer, R. D., Davies, J. K., et al. (EXPORT) 2002, A\&A, 384, 1038

Fisher, R. S., Telesco, C. M., Piña, R. K., Knacke, R. F., \& Wyatt, M. C. 2000, ApJ, 532, L141

Ghandour, L., Strom, S., Edwards, S., \& Hillenbrand, L. 1994 in The nature and evolutionary status of Herbig Ae/Be stars, ed. P. S. The, M. R. Perez, \& E. P. J. van den Heuvel, ASP Conf. Ser., 62, 223
Grady, C. A., Woodgate, B., Bruhweiler, F. C., et al. 1999, ApJ, 523, L151

Grady, C. A., Devine, D., Woodgate, B., et al. 2000, ApJ, 544, 895

Gray, R. O., \& Corbally, C. J. 1998, AJ, 116, 2530

Grinin, V. P., \& Rostopchina, A. N. 1996, ARep, 40, 171

Grinin, V. P., Shakhovskoi, D. N., Shenavrin, V. I., Rostopchina, A. N., \& Tambovtseva, L. V. 2002, ARep, 46, 646

Gullbring, E., Calvet, N., Muzerolle, J., \& Hartmann, L. 2000, ApJ, 544,927

Hartmann, L., Kenyon, S. J., \& Calvet, N. 1993, ApJ, 407, 219

Herbst, W., \& Shevchenko, V. S. 1999, AJ, 118, 1043

Hillenbrand, L. A., Strom, S. E., Vrba, F. J., \& Keene, J. 1992, ApJ, 397,613

Jayawardhana, R., Fisher, S., Hartmann, L., et al. 1998, ApJ, 503, L79

Johns, C. M., \& Basri, G. 1995, ApJ, 449, 341

Jura, M., Malkan, M., White, R., et al. 1998, ApJ, 505, 897

Kenyon, S. J., \& Hartmann, L. 1987, ApJ, 323, 714

Kurucz, R. L. 1993, ATLAS9 Stellar Atmosphere Programs and $2 \mathrm{~km} \mathrm{~s}^{-1}$ grid. CD-ROM No. 13, Cambridge, Massachusetts, Smithsonian Astrophysical Observatory

Li, A., \& Draine, B. T. 2001, ApJ, 554, 778

Li, A., \& Lunine, J. I. 2003, ApJ, 594, 987

Lorenzetti, D., Giannini, T., Nisini, B., et al. 2002, A\&A, 395, 637

Lowrance, P. J., Schneider, G., Kirkpatrick, J. D., et al. 2000, ApJ, 541,390

Lub, J., \& Pel, J. W. 1977, A\&A, 54, 137

Malfait, K., Bogaert, E., \& Waelkens, C. 1998, A\&A, 331, 211

Mannings, V. 1994, MNRAS, 271, 587

Mannings, V., \& Sargent, A. I. 1997, ApJ, 490, 792

Mannings, V., \& Sargent, A. I. 2000, ApJ, 529, 391

Mannings, V., Koerner, D. W., \& Sargent, A. I. 1997, Nature, 388, 555

Marsh, K. A., Silverstone, M. D., Becklin, E. E., et al. 2002, ApJ, 573, 425

Mayor, M., \& Queloz, D. 1995, Nature, 378, 355

Miroshnichenko, A., Ivezic, Z., \& Elitzur, M. 1997, ApJ, 475, 41; erratum in ApJ, 479, 153

Miroshnichenko, A., Ivezic, Z., Vinkovic, D., \& Elitzur, M. 1999, ApJ, 520,115

Miyake, K., \& Nakagawa, Y. 1993, Icarus, 106, 20

Mora, A., Merín, B., Solano, E., et al. (EXPORT) 2001, A\&A, 378, 116

Mouillet, D., Lagrange, A. M., Augereau, J. C., \& Ménard, F. 2001, A\&A, 372, L61

Muzerolle, J., D’Alessio, P., Calvet, N., \& Hartmann, L. 2003a, ApJ, in preparation

Muzerolle, J., Calvet, N., Hartmann, L., \& D’Alessio, P. 2003b, ApJ, in preparation

Natta, A. 1993, ApJ, 412, 761

Natta, A., Palla, F., Butner, H. M., Evans, N. J., II, \& Harvey, P. M. 1993, ApJ, 406, 674

Natta, A., Grinin, V. P., Mannings, V., \& Ungerechts, H. 1997, ApJ, 491,885

Natta, A., Grinin, V. P., \& Mannings, V. 2000, in Protostars and Planets IV, ed. V. Mannings, A. P. Boss, \& S. S. Russell (The University of Arizona Press), 559

Natta, A., Prusti, T., Neri, R., et al. 2001, A\&A, 371, 186

Oliveira, J.-M., Foing, B. H., van Loon, J. Th., \& Unruh, Y. C. 2000, A\&A, 362, 615

Oudmaijer, R. D., Palacios, J., Eiroa, C., et al. (EXPORT) 2001, A\&A, 379, 564

Penprase, B. E. 1992, ApJS, 83, 273

Pezzuto, S., Strafella, F., \& Lorenzetti, D. 1997, ApJ, 485, 290

Piétu, V., Dutrey, A., \& Kahane, C. 2003, A\&A, 398, 565 
Pollack, J. B., Hollenbach, D., Beckwith, S., et al. 1994, ApJ, 421, 615 Qi, C., Kessler, J., Koerner, D., et al. 2003, ApJ, submitted

Rieke, G. H., \& Lebofsky, M. J. 1985, ApJ, 288, 618

Schmidt-Kaler, T. 1982, Landolt-Börnstein New Series, 2B, Chapt. Physical parameters of the stars (New York: Springer-Verlag)

Shakura, N. I., \& Sunyaev, R. A. 1973, A\&A, 24, 337

Solano, E., González-Riestra, R., Talavera, A., et al. 2001, in Astronomical Data Analysis Software and Systems X, ed. F. R. Harnden, Jr., F. A. Primini, \& H. E. Payne, ASP Conf. Ser., 238, 152

Song, I., Caillaut, J.-P., Barrado y Navascués, D., \& Stauffer, J. R. 2001, ApJ, 546, 352

Steenman, H., \& Thé, P. S. 1991, Ap\&SS, 184, 9

Sylvester, R. J., Skinner, C. J., Barlow, M. J., \& Mannings, V. 1996, MNRAS, 279, 915

Sylvester, R. J., Dunkin, S. K., \& Barlow, M. J. 2001, MNRAS, 327, 133
Tambovtseva, L. V., \& Grinin, V. P. 2000, in Disks, Planetesimals and Planets, ed. F. Garzón, C. Eiroa, D. de Winter, \& T. Mahoney, ASP Conf. Ser., 219, 428

Testi, L., Natta, A., Shepherd, D. S., \& Wilner, D. J. 2001, ApJ, 554, 1087

Thi, W. F., Blake, G. A., van Dishoeck, E. F., et al. 2001, Nature, 409, 60

van den Ancker, M. E., de Winter, D., Tjin, A., \& Djie, H. R. E. 1998, A\&A, 330, 145

Waters, L. B. F. M., \& Waelkens, C. 1998, ARA\&A, 36, 233

Weinberger, A. J., Becklin, E. E., Schneider, G., et al. 1999, ApJ, 525, L53

Weinberger, A. J., Rich, R. M., Becklin, E. E., Zuckerman, B., \& Matthews, K. 2000, ApJ, 544, 937

Yi, S., Demarque, P., Kim, Y.-Ch., et al. 2001, ApJS, 136, 417

Zuckerman, B., Forveille, T., \& Kastner, J. H. 1995, Nature, 373, 494 